\title{
Solar wind plasma interaction with solar probe plus spacecraft
}

\author{
S. Guillemant ${ }^{1,2}$, V. Génot ${ }^{1}$, J.-C. Matéo-Vélez ${ }^{2}$, R. Ergun $^{3}$, and P. Louarn ${ }^{1}$ \\ ${ }^{1}$ IRAP (Institut de Recherche en Astrophysique et Planétologie), Université Paul Sabatier de Toulouse \& CNRS, \\ UMR5277, 9 avenue du Colonel Roche, BP 44346, 31028 Toulouse Cedex 4, France \\ ${ }^{2}$ ONERA (Office National d'Études et Recherches Aérospatiales), 2 avenue Édouard Belin, BP74025, \\ 31055 Toulouse Cedex 4, France \\ ${ }^{3}$ The Laboratory for Atmospheric and Space Physics, University of Colorado, Boulder, Colorado 80309, USA \\ Correspondence to: S. Guillemant (stanislas.guillemant@irap.omp.eu)
}

Received: 19 March 2012 - Revised: 26 June 2012 - Accepted: 29 June 2012 - Published: 24 July 2012

\begin{abstract}
D PIC (Particle In Cell) simulations of spacecraft-plasma interactions in the solar wind context of the Solar Probe Plus mission are presented. The SPIS software is used to simulate a simplified probe in the near-Sun environment (at a distance of $0.044 \mathrm{AU}$ or $9.5 R_{\mathrm{S}}$ from the Sun surface). We begin this study with a cross comparison of SPIS with another PIC code, aiming at providing the static potential structure surrounding a spacecraft in a high photoelectron environment. This paper presents then a sensitivity study using generic SPIS capabilities, investigating the role of some physical phenomena and numerical models. It confirms that in the near- sun environment, the Solar Probe Plus spacecraft would rather be negatively charged, despite the high yield of photoemission. This negative potential is explained through the dense sheath of photoelectrons and secondary electrons both emitted with low energies $(2-3 \mathrm{eV})$. Due to this low energy of emission, these particles are not ejected at an infinite distance of the spacecraft and would rather surround it. As involved densities of photoelectrons can reach $10^{6} \mathrm{~cm}^{-3}$ (compared to ambient ions and electrons densities of about $7 \times 10^{3} \mathrm{~cm}^{-3}$ ), those populations affect the surrounding plasma potential generating potential barriers for low energy electrons, leading to high recollection. This charging could interfere with the low energy (up to a few tens of eV) plasma sensors and particle detectors, by biasing the particle distribution functions measured by the instruments. Moreover, if the spacecraft charges to large negative potentials, the problem will be more severe as low energy electrons will not be seen at all. The importance of the modelling requirements in terms of precise prediction of spacecraft potential is also discussed.
\end{abstract}

Keywords. Interplanetary physics (Solar wind plasma) Space plasma physics (Electrostatic structures; Spacecraft sheaths, wakes, charging)

\section{Introduction}

Solar Probe Plus ( $\mathrm{SP}+)$ is a NASA mission which consists in studying the very close environment of the Sun (approaching as close as 9.5 solar radii above the Sun's surface). The in situ measurements and imaging will help to understand the heating process of the Sun corona and the acceleration of the solar wind. The launch is planned for 2018 and the first perihelion in 2021. At such distances from the Sun, the expected environment of Solar Probe Plus should be quite hot and dense, leading the spacecraft and its on board instruments to suffer from high temperatures, charging and erosion. In particular, estimations of the satellite potential behaviour in such plasmas are important to predict the possible biases on plasma and electric measurements. Furthermore, the satellite velocity combined to the relative speed of the solar wind will create an ion wake which will likely increase the disturbances on the near probe environment ${ }^{1}$. In a similar context but with less extreme conditions; the Solar Orbiter spacecraft will reach regions further from the Sun $(\sim 0.28 \mathrm{AU})$. The impact of such conditions will be studied in a further paper.

Following observations of recollected photoelectrons and secondary electrons on the ATS 6 spacecraft, Whipple Jr. (1976) developed a theory for a spherically symmetric photoelectron sheath, including effects of ions, thermal electrons and secondaries. The aim was to determine whether the potential barrier responsible for the secondaries reflection

\footnotetext{
${ }^{1}$ Solar Probe website, http://solarprobe.jhuapl.edu/
} 
was originating from those same particles or not. However, a comparison with the spacecraft data showed that the observed barrier of potentials is too large to be explained by the model (i.e. a spherically symmetric photoelectron or secondary electron sheath surrounding a uniformly charged spacecraft), and the authors concluded that the most probable explanation is that some portions of the ATS 6 surfaces are charged to different potentials. Actually, this thick sheath approximation is valid for large Debye lengths of emitted electrons, which is not the case for regions as close as $0.044 \mathrm{AU}$ to the Sun. The Debye lengths of all secondaries in this nearSun environment barely exceed a few centimetres, far from being of the order of the Solar Probe Plus dimensions. Thus the model of Whipple Jr. (1976) is not applicable in the present study.

Following Whipple's analysis and in the context of instruments for active control of the potential, Zhao et al. (1996) proposed an analytical approach to compute the electrostatic barrier and compared to Geotail measurements. However, this analysis is also only relevant in the sheath approximation and does not consider the secondary electronic emission.

Referring to the Helios spacecraft, a paper (Isensee, 1977) presents particle-in-cell simulations of the plasma environment of a spacecraft in the Solar wind, at $0.2 \mathrm{AU}$ from the Sun. Using a certain number of discrete particles, injected at the boundaries of a simulation box with the appropriate distributions, the code moved them in the potentials and calculated the local densities from the number of particles per cell of a mesh. The potential was updated at the next time step by solving Poisson's equation. A two-dimensional model for numerical plasma simulation $(9 \mathrm{~m} \times 19.75 \mathrm{~m}$ domain, divided into $0.25 \times 0.25 \mathrm{~m}$ cells) with a simplified probe geometry was used. With a conducting spacecraft, the consideration of $1 \mathrm{eV}$ mean energy photoelectrons and the expected Solar wind conditions, the author obtained a lightly positively charged satellite $(+2.9 \mathrm{~V})$ surrounded by negative plasma potentials in the wake and in the ram. In front of the sunlit face, due to very high densities of photoelectrons, the local potential reached $-1.4 \mathrm{~V}$ and in the ion wake behind the probe: $-4.5 \mathrm{~V}$. The $1 \mathrm{eV}$ emitted photoelectrons are thus recollected by the surfaces of the probe. The rest of the paper focusses on the consequences in distortions of measured electron distribution functions. Such simple simulations of photoelectron clouds and their effects on spacecraft charging were already of interests for the understanding of plasma measurements disturbances. In these simulations, the secondary electronic emission was not modelled. We can thus easily imagine that with an extra secondary electron cloud surrounding the spacecraft and with a more energetic and concentrated environment that exists closer to the Sun, these simulated effects would be amplified.

Thiébault et al. (2004) studied the potential barrier in the electrostatic sheath around a magnetospheric spacecraft, for cases of conductive spacecraft like Geotail and Cluster. A fully self-consistent analytical model of the plasma around an electron emitting central body in a spherically symmetric geometry was used to analyse the electrostatic sheath around an idealized spacecraft. It was shown by comparison with 3D PIC simulations that non-monotonic potential with negative potential barrier can exist all around a positively charged spacecraft (with Debye length of the order of the central body radius or more) even in the case of an asymmetric illumination pattern. Those existing potential barriers at distances from the Sun of $1 \mathrm{AU}$ encourage advanced studies for nearSun environment conditions (where even stronger barriers may exist): the preparation of the Solar Probe Plus mission that may be affected by such potential barriers has naturally been a motivation to perform such study.

Lipatov et al. (2010) studied the interactions of the solar wind with SP+ through 3-D hybrid simulations at a distance of $9.5 R_{\mathrm{S}}$. Their simulations are focused on the electric and magnetic fields surrounding the spacecraft. They do not take into account the spacecraft charging, the charge separation effects, the electron dynamics near the spacecraft, or the effects due to photoionization and electron impact ionization near the spacecraft. They demonstrated that the current closure near the spacecraft is very complicated and is directed by the external magnetic field. Some magnetic field barrier forms at the front of the heat shield, whereas strong whistler/Alfvén waves form in both upstream and downstream regions. The values of the electric field oscillations near the spacecraft bus may be of the same order as the maximum of expected electric field at an antenna. Simulated electric field perturbations are comparable to or exceed the maximum electric field expected for the SP+ spacecraft.

Also in the Solar Probe Plus context at 0.044 AU from the Sun, simulation results provided in Ergun et al. (2010) show that a negatively charged satellite is obtained using a PIC code and a simplified geometry model. High potential barriers for emitted photoelectrons and secondary electrons appear in the ram and the wake sides of the probe, due to their high densities in these regions, and make those low energy particles recollected by the spacecraft. The balance of currents is obtained for a negative spacecraft potential. We will cross-compare our numerical tool with the code used in Ergun et al. (2010) and a description of this model is given in Sect. 2.1, the corresponding results are presented in Sect. 2.2.

The simulation tool used in this study is SPIS, a software development project of the European Space Agency (ESA). It is developed as an open source and versatile code with the support of the Spacecraft Plasma Interaction Network in Europe (SPINE) community ${ }^{2}$. The first development phase of the project has been performed by ONERA/DESP, Artenum and University Paris VII (through the ESA contract Nbr: 16806/02/NL/JA). Some developments were funded by the French space agency (CNES). It is a simulation software based on an electrostatic 3-D unstructured particle-in-cell plasma model and consisting of a

\footnotetext{
${ }^{2}$ SPIS web site, http://dev.spis.org/projects/spine/home
} 
JAVA based highly modular object oriented library, called SPIS/NUM. More accurate, adaptable and extensible than the existing simulation codes, SPIS is designed to be used for a broad range of industrial and scientific applications. The simulation kernel is integrated into a complete modular pre-processing/computation/postprocessing framework, called SPIS/UI, allowing a high degree of integration of external tools, such as CAD, meshers and visualization libraries (VTK), and a very easy and flexible access to each level of the numerical modules via the Jython script language. Developed in an open source approach and oriented toward a community based development, SPIS is available for the whole community and is used by members of the European SPINE network. SPIS should address a large majority of the new challenges in spacecraft plasma interactions, including the environment of electric thruster systems, solar arrays plasma interactions, and modelization of scientific plasma instruments.

The numerical core and the user interface have been developed by ONERA and the Artenum company, respectively (Roussel et al., 2008a). Recent enhancements have consisted in improving multi time scale and multi physics capabilities (Roussel et al., 2012). One first paper on a real engineering application, SMART-1 by Hilgers et al. (2006), studied the electrostatic potential variation of the probe and the first SPIS validations by comparison with theoretical models are presented in Hilgers et al. (2008). The effect of inorbit plasma on spacecraft has been modelled in a wide range of configurations: geosynchronous (GEO) spacecraft charging (Roussel et al., 2012), electric propulsion (Roussel et al., $2008 \mathrm{~b}$ ), barrier of potential at millimetre scale (Roussel et al., 2008a) and electrostatic discharge onset on GEO solar panels (Sarrailh et al., 2010). The ONERA plasma chamber, named JONAS, was simulated and the results compared to experiments in Matéo-Vélez et al. (2008). It has also been compared with other numerical models (Roussel et al., 2012; Matéo-Vélez et al., 2012a).

The objective of this paper is to estimate the disturbances on near-Sun probe measurements using the SPIS software. Section 2 presents the cross-comparison of SPIS software and the code described in Ergun et al. (2010). Results using SPIS with more complete modelling and a parametric study are described in Sect. 3. Conclusion and perspectives are presented in Sect. 4.

\section{Cross-comparison of the two codes}

In this section, we present a simplified model of Solar Probe Plus in a near-Sun environment using two codes: SPIS and the code described in Ergun et al. (2010). It aims at crosscomparing these codes with an identical set of parameters. We describe first the approach used in both codes in Sect. 2.1 and provide the results in the next one.

\subsection{Models}

The comparison is performed on the same case as in Fig. 5 of Ergun et al. (2010). The corresponding code is used here with modifications regarding the previously published paper. The mesh has been refined $(1 \mathrm{~cm}$ instead of $2 \mathrm{~cm})$ and the photoemission has been changed (maxwellian photoelectrons of temperature $3 \mathrm{eV}$ instead of the double Maxwellian of temperatures $2.7 \mathrm{eV}$ and $10 \mathrm{eV}$ ). Concerning the SEEY (Secondary Electronic Emission Yield) it is assumed to be equal to 1 , instead of the BeCu SEEY properties reported previously in Ergun et al. (2010) (referenced in Lai, 1991). A higher order calculation of thermal electron trajectories has been implemented and finally, the scattered thermal electrons $(15 \%)$ that were not included in the electron density calculation are now taken into account. This simulation is referred to as simulation A in the following. For the sake of completeness, we remind the reader of some details in the following paragraph.

This code is a Poisson solver and electron tracing program considering a 3-D cylindrically symmetric domain on a two dimensional (2-D) grid. The numerical architecture has two parts, which (1) determine the potential structure $(\phi)$ surrounding the spacecraft via a Poisson solver (given a charge distribution) and (2) determine the charge distribution via particle tracing performed in 3-D (given $\phi$ ). The domain is a $5 \mathrm{~m}($ in $R) \times 10 \mathrm{~m}$ (in $X$ ) cylinder with $500 \times 1000$ 2-D grid. The grid spacing is $1 \mathrm{~cm}$ in both $X$ and $R$. The space environment is taken from Lipatov et al. (2010) and Ergun et al. (2010) and presented in Table 1. The spacecraft is assumed to be a fully conducting cylinder, $1 \mathrm{~m}$ in radius and $2 \mathrm{~m}$ long, with one end allowed to emit photoelectrons. Ambient electrons follow a Maxwell-Boltzmann equilibrium distribution. Ion drift velocity is $-300 \mathrm{~km} \mathrm{~s}^{-1}$ in the direction down the $\mathrm{z}$-axis that reproduces a Solar wind bulk speed estimated at $200 \mathrm{~km} \mathrm{~s}^{-1}$ from the Sun, added to the relative probe velocity of $100 \mathrm{~km} \mathrm{~s}^{-1}$ toward the Sun. Ion modelling is very simple since it is assumed that their density is uniform, except behind the cylinder (in the -z direction) where their density is null. $10^{6}$ photoelectrons are randomly created on the sunlit surfaces, along isotropic directions, with a Maxwellian energy distribution and a $3 \mathrm{eV}$ mean energy (Ergun et al., 2010; Pedersen, 1995). A rather high photoelectron current at $1 \mathrm{AU}$ of $J_{\mathrm{ph}}$ of $57 \mu \mathrm{A} \mathrm{m}^{-2}$ is scaled to the position of the spacecraft $(0.044 \mathrm{AU})$, giving an injected current density $J_{\mathrm{ph}}$ of $29 \mathrm{~mA} \mathrm{~m}^{-2}$. The Debye length $\lambda_{\mathrm{ph}}$ for photoelectrons is $\sim 3 \mathrm{~cm}$. Secondary electron emission under ion impact (SEI) efficiency is assumed arbitrarily to be $100 \%$ (each impacting ion liberates one secondary electron). Secondary electron emission under electron impact (SEE) is modelled by creating electrons randomly over the spacecraft surfaces with a $2 \mathrm{eV}$ characteristic energy. The thermal efficiency $\epsilon_{\text {the }}$ (i.e. the fraction of electrons that strike the surface and are absorbed) arbitrarily equals to 0.85 . Those that are not absorbed (0.15) are assumed to be scattered without 
Table 1. Input parameters for the cross-comparison test case.

\begin{tabular}{|c|c|c|c|}
\hline Parameter & $\begin{array}{l}\text { Value } \\
\text { (Ergun et al., 2010) }\end{array}$ & $\begin{array}{l}\text { Value (R. E. Ergun, unpublished data, 2012) } \\
\text { Simulation A }\end{array}$ & $\begin{array}{l}\text { Value (SPIS) } \\
\text { Simulation B }\end{array}$ \\
\hline Thermal electron/ion density $n_{\mathrm{e}, \mathrm{i}}$ & $7 \times 10^{9} \mathrm{~m}^{-3}$ & $7 \times 10^{9} \mathrm{~m}^{-3}$ & $7 \times 10^{9} \mathrm{~m}^{-3}$ \\
\hline Thermal electron temperature $T_{\mathrm{e}}$ & $85 \mathrm{eV}$ & $85 \mathrm{eV}$ & $85 \mathrm{eV}$ \\
\hline Electron modelling & Maxwell-Boltzmann & Maxwell-Boltzmann & Maxwell-Boltzmann \\
\hline Ion modelling & $\begin{array}{l}\text { uniform } \\
\text { (null in wake) }\end{array}$ & $\begin{array}{l}\text { uniform } \\
\text { (null in wake) }\end{array}$ & $\begin{array}{l}\text { PIC } \\
\text { (with no deflection) }\end{array}$ \\
\hline Ion temperature $T_{\mathrm{i}}$ & N/A & N/A & $0.01 \mathrm{eV}$ \\
\hline Ion velocity & $V_{Z}=-300 \mathrm{~km} \mathrm{~s}^{-1}$ & $V_{Z}=-300 \mathrm{~km} \mathrm{~s}^{-1}$ & $V_{Z}=-300 \mathrm{~km} \mathrm{~s}^{-1}$ \\
\hline Ion type & $H^{+}$ & $H^{+}$ & $H^{+}$ \\
\hline Material & Conductive & Conductive & Conductive \\
\hline Photoelectron temperature $T_{\mathrm{ph}}$ & $\begin{array}{l}95 \% \text { at } 2.7 \mathrm{eV} \\
+5 \% \text { at } 10 \mathrm{eV}\end{array}$ & $3 \mathrm{eV}$ & $\begin{array}{l}3 \mathrm{eV} \\
(+ \text { case with } 10 \mathrm{eV})\end{array}$ \\
\hline$J_{\mathrm{ph}}\left(\mathrm{mA} \mathrm{m}^{-2}\right)$ & 29 & 29 & 29 \\
\hline SEE Distribution & maxwellian, $2 \mathrm{eV}$ & maxwellian, $2 \mathrm{eV}$ & maxwellian, $2 \mathrm{eV}$ \\
\hline True Secondary Emission Yield & $\begin{array}{l}\text { curves of } \mathrm{BeCu} \\
\text { in Lai }(1991)\end{array}$ & 1 & 2.47 \\
\hline Backscattered Electron Yield & 0.15 & 0.15 & 0.17 \\
\hline Meshing & $2 \mathrm{~cm}$ & $1 \mathrm{~cm}$ & from 2 to $50 \mathrm{~cm}$ \\
\hline External boundary conditions & $0 \mathrm{~V}$ & $0 \mathrm{~V}$ & $0 \mathrm{~V}$ \\
\hline
\end{tabular}

energy loss. The yield of electron secondary emission under absorbed electrons is arbitrarily 1 . The potential at the limits of the simulation box is set at $0 \mathrm{~V}$.

SPIS uses an unstructured tetrahedral mesh that allows it to refine spatial resolution near regions of interest. The plasma model treats ions fully kinetically with realistic masses. Electrons can be treated fully kinetically (full PIC) or as a fluid, like in the Maxwell-Boltzmann statistics equilibrium model approximation. A multi-zone modelling combines fluid and PIC description of electrons. Particle sources from ambient environment are modelled by a Maxwellian distribution for electrons and ions (a drift can be added for ions); up to two populations per species can be considered. The electric field is computed from a finite element discretization of Poisson's equation and solved with an iterative conjugate gradient solver. An implicit Newton-type solver is used for a non-linear Poisson equation, in the case of Boltzmann distributions for ambient electrons. The boundary condition on an external boundary is a mixed Dirichlet-Neumann. The boundary condition on a spacecraft is Dirichlet and based on the spacecraft surface potential evolution. Finally, it handles spacecraft geometrical singularities (wires, plates) by extracting the singular part of the field. The charge exchange volume interaction is modelled by a Monte Carlo model. The spacecraft material properties considered are: secondary emission (under electron/proton/UV), conductivities (surface/volume, intrinsic/radiation induced), electron field emission, sputtering (recession rate, product generation and transport). The spacecraft equivalent circuit is composed of dielectric coatings, user-defined discrete components and is solved using an implicit solver, with auto-adaptive time step.

In the SPIS simulation, referred to as simulation $B$ in the following, the 3-D domain is $5 \mathrm{~m}$ (in $R$ ) $\times 10 \mathrm{~m}$ (in $X$ ), with a progressive refinement of the mesh (until $2 \mathrm{~cm}$ on the sunlit face of the cylinder); see Fig. 1 showing the Gmsh model for the satellite (Gmsh is an automatic 3-D finite element mesh generator with build-in pre- and post-processing facilities). An intermediate cylinder has been created to limit a fast enlargement of the mesh near the satellite. It is forced to have a $15 \mathrm{~cm}$ grid spacing on the sun side and $30 \mathrm{~cm}$ on the other side. This intermediate cylinder has no physical existence, its aim is to control the meshing growth. The input parameters are presented in Table 1 in comparison to those used with the other code. Some parameter differences exist. First, the generic PIC (Particle In Cell) modelling used in SPIS was adapted for ions in order to fit the modelling used in the other code: ions are emitted at the boundary with a velocity of $-300 \mathrm{~km} \mathrm{~s}^{-1}$, a temperature of $0.01 \mathrm{eV}$ and they are not deflected. Second, the material used has complete curves of SEE yield and backscattering yield versus incident electron energy, see Fig. 2. For the isotropic ambient electron with energy of $85 \mathrm{eV}$, the backscattering yield is 0.17 and the true secondary emission yield is 2.47 . So comparing to the simulation with the other code, this SPIS simulation will generate more secondary electrons. Third, no SEI is modelled.

Plasma frequency associated to thermal and photoelectrons are $748 \mathrm{kHz}$ and $2069 \mathrm{kHz}$, respectively. Debye length associated to ambient and photo electrons are $80 \mathrm{~cm}$ and $3 \mathrm{~cm}$, respectively. That means that photoelectrons should rule the plasma behaviour around the satellite, at least on the 


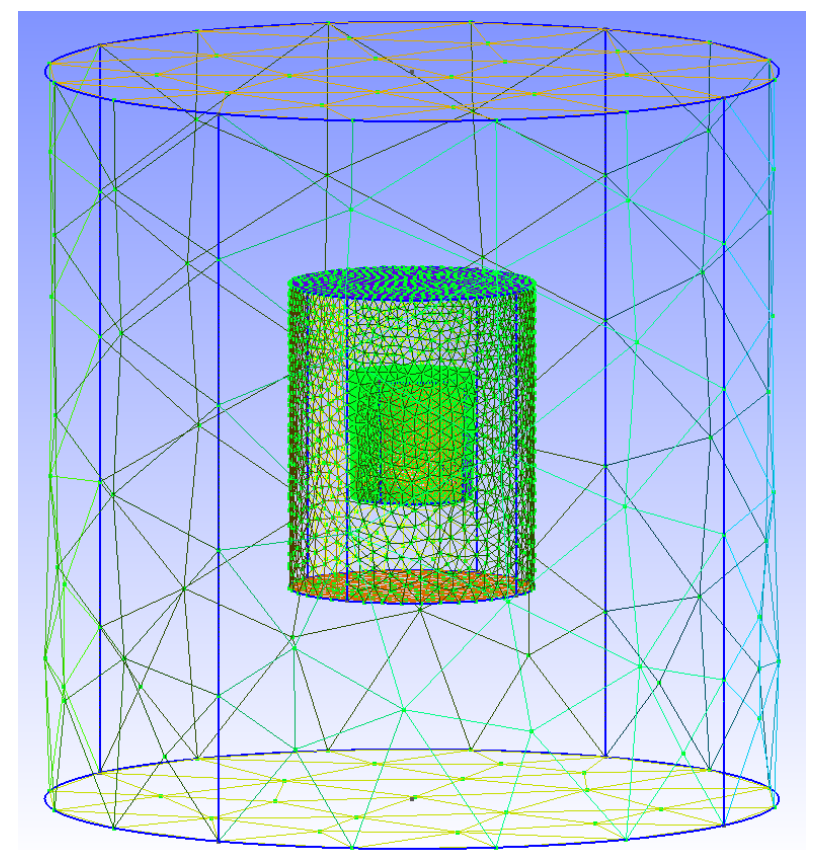

Fig. 1. The GMSH model of the simplified Solar Probe Plus spacecraft used in SPIS simulations with the associated meshing grid. The $\mathrm{z}$-axis described in the text is the vertical axis in this figure. From the outside to the inside the boundary of the simulation box, the intermediate meshing cylinder (which has no physical meaning) and the satellite cylinder can be distinguished.

sun-side face. This induces the necessity to have very small grids and time steps. The simulation duration is set to $40 \mu \mathrm{s}$, with time steps of $50 \mathrm{~ns}$.

\subsection{Results}

Results obtained with simulation A are presented in Fig. 3. Results are qualitatively comparable to those previously obtained in the Fig. 5 of Ergun et al. (2010), except that the spacecraft floats at $-15.5 \mathrm{~V}$ instead of $-4.15 \mathrm{~V}$. This is mainly due to the refined mesh used to simulate the secondary electron barrier, the changes in the SEEY and the consideration of the scattered electrons in the electron density calculation (see Sect. 2.1). Those changes deepened the barrier around the satellite and caused a significant change in the floating potential.

Table 2 shows all net currents on the spacecraft. Concerning simulation $\mathrm{A}$, the total thermal electron current arriving on the spacecraft $I_{\text {the }}$ reaches $-25.6 \mathrm{~mA}$. Due to $\epsilon_{\text {the }}$ (i.e. the fraction of electrons that strike the surface and are absorbed), which equals $85 \%$, there are $-21.8 \mathrm{~mA}$ effectively absorbed by the structure and $-3.8 \mathrm{~mA}$ of electron current that is backscattered without energy loss. For secondary electron currents, the account leads then to $21.8 \mathrm{~mA}$ emitted, $3.8 \mathrm{~mA}$ backscattered, $1.0 \mathrm{~mA}$ due to ion impact, and $-10.2 \mathrm{~mA}$ rec-
Table 2. Comparison of net currents on spacecraft for simulations $\mathrm{A}$ and $\mathrm{B}$.

\begin{tabular}{lrr}
\hline Net current $(\mathrm{mA})$ & $\begin{array}{r}\text { Simulation A } \\
\text { SC at }-15.5 \mathrm{~V}\end{array}$ & $\begin{array}{r}\text { Simulation B (SPIS) } \\
\text { SC at }-20 \mathrm{~V}\end{array}$ \\
\hline$I_{\text {the }}$ & -25.6 & -25.2 \\
$I_{\text {ion }}$ & 1.0 & 0.7 \\
$I_{\mathrm{ph}}$ & 8.1 & 6.6 \\
$I_{\mathrm{se}}$ & 16.4 & 17.7 \\
\hline$\sum I$ & -0.1 & -0.2 \\
\hline
\end{tabular}

ollected, giving a net current $I_{\mathrm{se}}$ of $16.4 \mathrm{~mA}$. The net current for photoelectrons equals $8.1 \mathrm{~mA}$.

Results obtained in simulation B with SPIS are qualitatively the same, with the formation of a photoelectron barrier and a negative spacecraft, floating at $-20 \mathrm{~V}$. Figure 4 shows the evolution of currents on the spacecraft and of the surface potential versus time. After a transient regime, where the potential grows until $3.9 \mathrm{~V}$ due to strong photoemission, the spacecraft then reduces to a permanent $-20 \mathrm{~V}$ voltage. At that time, collected and emitted currents are balanced.

The collected currents from thermal electrons and ions reach $-25.2 \mathrm{~mA}$ and $1.0 \mathrm{~mA}$, respectively. The photoelectronic emission is constant over time ( $-91 \mathrm{~mA}$ during a constant solar flux) while the emission of secondary electrons depends on the spacecraft potential: when $\phi_{\mathrm{SC}}$ is highly positive at the first steps of the simulation, the structure collects a high current density of thermal electrons and emits consequently many secondary electrons. Once the spacecraft potential reached equilibrium, the emitted current from secondary electrons sets to a value of $105.3 \mathrm{~mA}$. Large recollected currents of SEE and photo electrons $(-87.5 \mathrm{~mA}$ and $-84.4 \mathrm{~mA}$, respectively) are observed. Thus, those two last populations have a larger impact than the ambient plasma populations. The net SEE current $I_{\mathrm{se}}$ is $17.7 \mathrm{~mA}$ and the net photoelectron current $I_{\mathrm{ph}}$ is $6.6 \mathrm{~mA}$. All net current values are visible in Table $2.83 \%$ of emitted secondary electrons are recollected and this ratio goes up to $93 \%$ for photoelectrons, even if the spacecraft is negative. This is a clear effect of potential barriers represented in Fig. 5, that shows the plasma potential around the spacecraft.

Figure 6 indicates that the ram barrier has a dimension of a few $\mathrm{cm}$, which correctly fits with $\lambda_{\mathrm{ph}}=3 \mathrm{~cm}$ and $\lambda_{\mathrm{se}}=6 \mathrm{~cm}$ and a height of $-11 \mathrm{~V}(-20 \mathrm{~V}$ on SC compared with $-31 \mathrm{~V}$ at the barrier maximum). The wake barrier is larger $(1 \mathrm{~m})$ due to the absence of ions in this region. The potential barrier is $-25 \mathrm{~V}(-45 \mathrm{~V}$ at the barrier maximum). Figure 7 reveals the existence of a $-4 \mathrm{~V}$ potential barrier facing the side of the cylinder. The isocontour line at $-20 \mathrm{~V}$ is $60 \mathrm{~cm}$ from the spacecraft in the $\mathrm{x}$-direction. This potential barrier leads to SEE electrons recollection too.

Concerning populations, both simulations A and B exhibit the same global behaviour, as seen in Fig. 3 and in Fig. 8. 


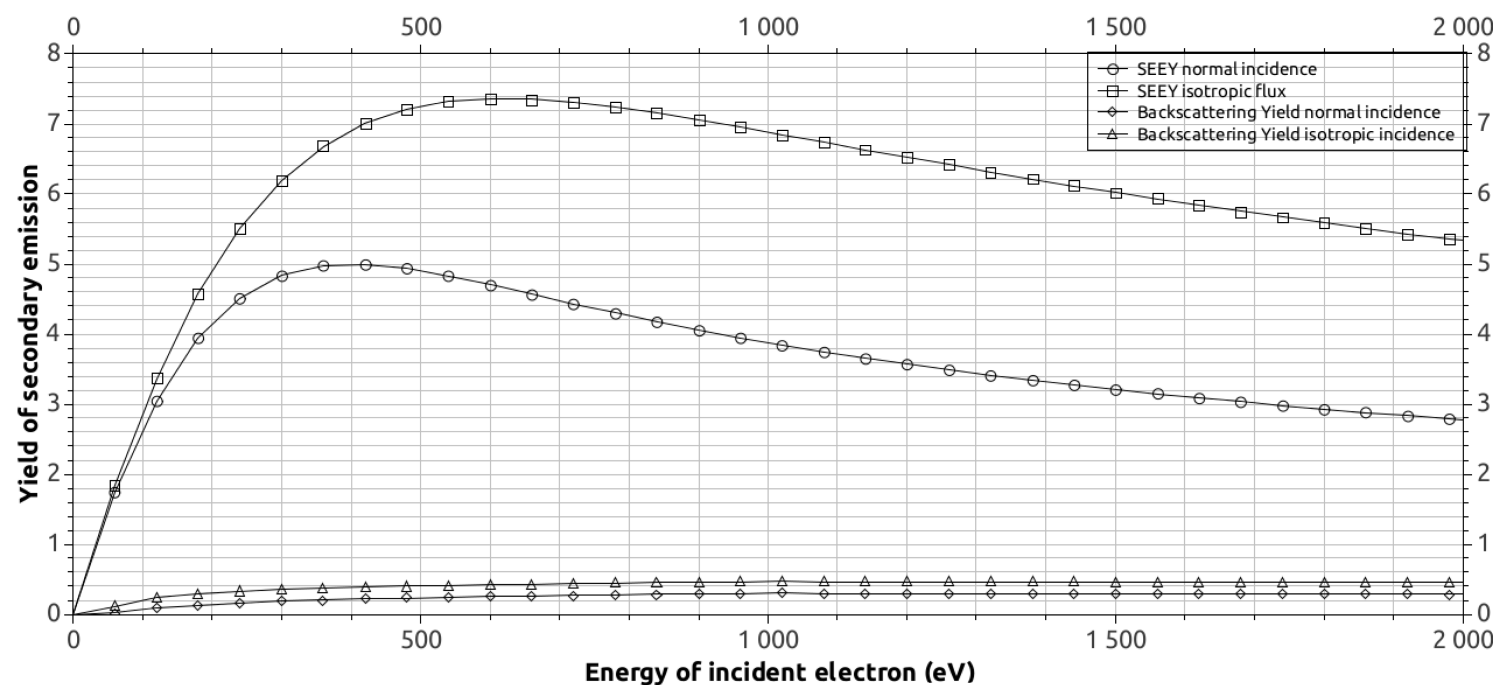

Fig. 2. Cross comparison simulation B (SPIS): Secondary Electron Emission Yield (SEEY) and the backscattering yield vs. incident electron energy (with a normal or isotropic incoming flux).

The diminution of the electron density due to the high negative potential from the structure and the potential barriers (at the front and the back of the cylinder) fits the Boltzmann distribution used. The ion wake is similarly solved, with a small ion non-null thermal velocity effect in simulation B with SPIS.

The most dense population of plasma are photoelectrons, emitted here from the sunlit face at densities of about $10^{11} \mathrm{~m}^{-3}$ and they are spreading around as far as the wake zone. As they are emitted from the sunlit face and depending on their energies and potential barriers, photoelectrons can move as far as the side of the cylinder that explains the density of particles in this region even though this circular face does not emit photoelectrons. The highly negative potential present in the wake prevents photoelectrons from penetrating this area.

The same effect of a near-sun environment on a spacecraft appears: its structure tends to settle at a negative potential, due to surrounding electrostatic barriers that originate from secondary electrons and photoelectrons. In the wake, negative potentials are somewhat different: $-32 \mathrm{~V}$ and $-45 \mathrm{~V}$ in simulation $\mathrm{A}$ and $\mathrm{B}$, respectively. At the front, $-22 \mathrm{~V}$ and $-31 \mathrm{~V}$ maximum potentials are obtained in simulation $\mathrm{A}$ and B, respectively. However, looking at the effective potential barrier values, simulation $B$ results in ram and wake barriers of $-11 \mathrm{~V}$ and $-25 \mathrm{~V}$ while simulation $\mathrm{A}$ results in $-7 \mathrm{~V}$ and $-17 \mathrm{~V}$. Furthermore, comparing the plasma potential maps for both cases, simulation B provides a more developed potential barrier on the side of the cylinder. This seems to be a direct effect of the higher SEY used in simulation B (2.47 instead of 1). In Fig. 8, SEE electron density of $\sim 10^{11} \mathrm{~m}^{-3}$ is observed over all surfaces of the spacecraft. It is large compared to that of thermal electrons around the cylinder $\left(5.5 \times 10^{9} \mathrm{~m}^{-3}\right)$. In that case, a potential barrier due to SEE electrons $(-4 \mathrm{~V})$ is added to that of photoelectrons. Density values above those faces and especially in the wake are the lowest $\left(10^{6} \mathrm{~m}^{-3}\right)$. It is through the sides of the cylinder that SEE electrons mostly escape.

All net currents are similar, except a $2 \mathrm{~mA}$ gap for photoelectron currents $(8.1 \mathrm{~mA}$ and $6.6 \mathrm{~mA}$ for simulation A and $\mathrm{B}$, respectively) due to a higher front potential barrier. This difference may be assigned to differences in meshing or photoelectrons dynamics. Concerning the SEE electron current, net values are also similar (16.4 and $17.7 \mathrm{~mA}$ for simulation $\mathrm{A}$ and $\mathrm{B}$, respectively), but the main difference lies in the emitted current: $I_{\text {the }}=-25.2 \mathrm{~mA}$ and $I_{\mathrm{se}}(\mathrm{emitted})=105.3 \mathrm{~mA}$ for SPIS simulation B, and $I_{\text {the }}=$ $-25.6 \mathrm{~mA}$ and $I_{\mathrm{se}}$ (emitted) $=21.8 \mathrm{~mA}$ for simulation A. The higher emission within SPIS leads to higher potential barriers facing the entire spacecraft surface which become locally one dimensional. Thus, a higher recollection rate of $83 \%$ is obtained. In simulation A, SEE electrons have more opportunities to escape through the side of the cylinder as in this region the potential barrier is visibly thinner (explaining a recollection rate of $45 \%$ ). Furthermore, the current $I_{\mathrm{se}}$ gathers both secondary and backscattered electrons. Through a certain yield depending on the incident particle energy, the SPIS backscattered particles will get out of the structure with $2 / 3$ of their initial energy (regarding to the other code where the backscattered keep all their energy). In SPIS simulation $\mathrm{B}$, the recollection of the backscattered is thus higher.

Lastly, in simulation A, it is assumed that $100 \%$ of impacting ions emit one secondary electron, which has however only a small impact on the total emitted current.

Finally, given the differences of modelling of the 2 simulations, the results are in good agreement. Possible negative charging of spacecraft in near-Sun conditions is obtained in this cross-comparison study. 

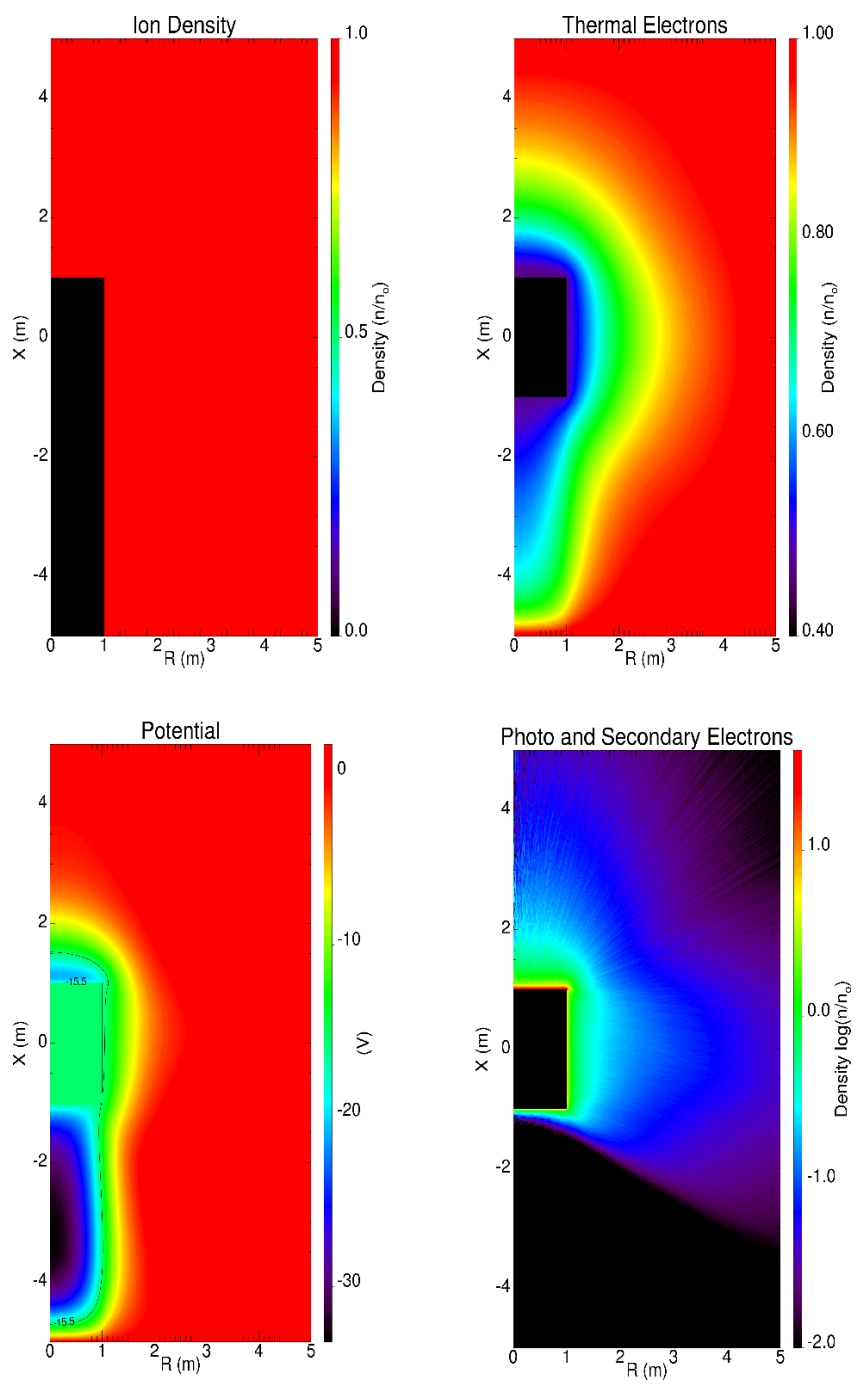

Fig. 3. Cross comparison simulation A. From the upper left to the bottom right of the picture: Ion density; Thermal electron density; SC potential. Photoelectron and SEE densities. $n_{0}$ is the plasma density: $7.10^{9} \mathrm{~m}^{-3}$.

\subsection{Photoelectron energy}

As discussed previously, the photoelectron and SEE electron models impact a lot the spacecraft potential. In this paragraph, a simulation $\mathrm{C}$ is run using SPIS in the same configuration as in simulation $\mathrm{B}$, except the photoelectron temperature of $10 \mathrm{eV}$ (instead of $3 \mathrm{eV}$ ). The spacecraft potential is now $-9.7 \mathrm{~V}$, explained by the fact that photoelectrons have more energy to spread further and escape the ram potential barrier, see Fig. 9. The position of the barrier gets further from the surface, as seen on Fig. 6. The barrier structure possibly gets closer to a three dimensional barrier compared to the previous case (almost one dimensional). The amount of photoelectrons emitted is the same but the position of the
Table 3. Parameters used for the nominal simulation S1.

\begin{tabular}{ll}
\hline Parameter & Value \\
\hline Thermal electron density $n_{\mathrm{e}}$ & $7 \times 10^{9} \mathrm{~m}^{-3}$ \\
Thermal electron temperature $T_{\mathrm{e}}$ & $85 \mathrm{eV}$ \\
Ion density $n_{\mathrm{i}}$ & $7 \times 10^{9} \mathrm{~m}^{-3}$ \\
Ion temperature $T_{\mathrm{i}}$ & $82 \mathrm{eV}$ \\
Ion type & $H^{+}$ \\
Ion modelling & $\mathrm{PIC}$ \\
Backscattered electron & Active \\
Photoelectron temperature $T_{\mathrm{ph}}$ & $3 \mathrm{eV}$ \\
Debye length for thermal electrons $\lambda_{\text {the }}$ & $0.8 \mathrm{~m}$ \\
Material & Conductive \\
Meshing & from $5 \mathrm{~cm}$ to $2 \mathrm{~m}$ \\
Number of tetrahedrons & $\sim 158000$ \\
External boundary conditions & Fourier: $1 / r^{2}$ \\
& decrease of potential \\
\hline
\end{tabular}

maximum is further. It has a strong impact close to the side of the sunlit disk.

\section{Parametric study using SPIS}

The need for complementary simulations comes from three points: (1) the necessity of a full PIC description of the environment, (2) the uncertainty on the secondary electron emission (linked to the chosen material coating the probe), and (3) an ion temperature more relevant to the one expected at this distance to the Sun.

In this section, SPIS capabilities are used to simulate the same near-Sun environment with the same spacecraft geometry as in simulation B to perform a parametric study both on numerical and physical parameters. The model and simulations are defined in Sect. 3.1, the results in Sect. 3.2.

\subsection{Model}

The same geometry is used as in Fig. 1, except a larger external box of dimensions $R=6 \mathrm{~m}$ and $X=12 \mathrm{~m}$. The grid spacing is $5 \mathrm{~cm}$ on the sunlit face (toward the z-axis), $15 \mathrm{~cm}$ on the other side and $2 \mathrm{~m}$ all over the domain limits. The whole meshed volume contains $\sim 158000$ tetrahedrons. The same environment is used. However, the genericity of SPIS permits to change the hybrid model (PIC for ions and Maxwell Boltzmann for electrons) to full PIC. It must be noticed that the Maxwell-Boltzmann distribution is exact when the satellite potential is negative and if no potential barrier exists. The Boltzmann model is only approximate if a potential barrier exists and is more negative: the less energetic electrons of the distribution should not be able to cross this barrier, so the Boltzmann model is overestimating the particles arriving on the satellite. Of course, it becomes completely wrong if potential barriers are large or if the spacecraft is significantly positive. In the simulations presented below, the full-PIC 

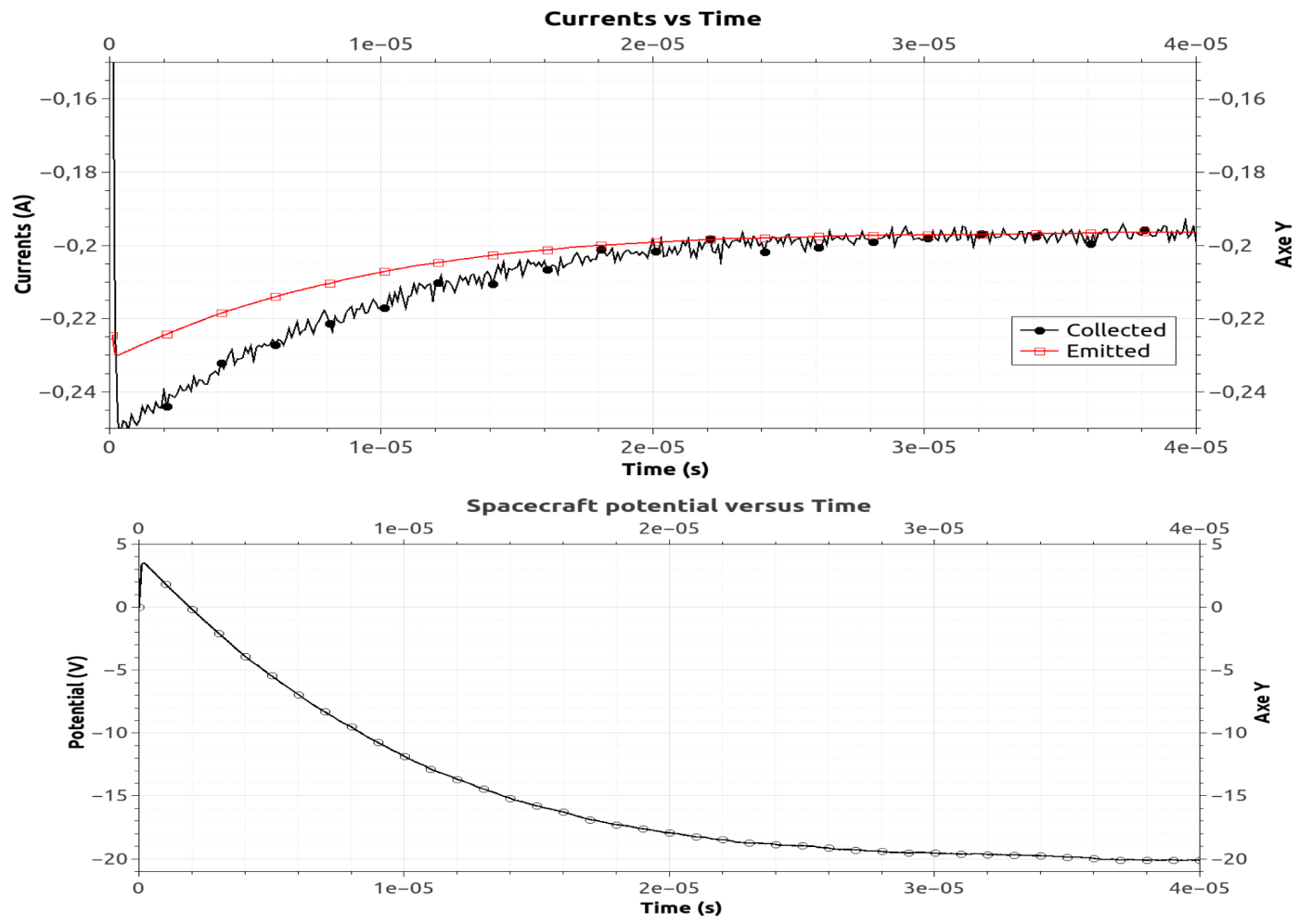

Fig. 4. Cross comparison simulation B (SPIS). Evolution versus time of all collected and emitted currents (on top) and of spacecraft average surface potential (on the bottom).

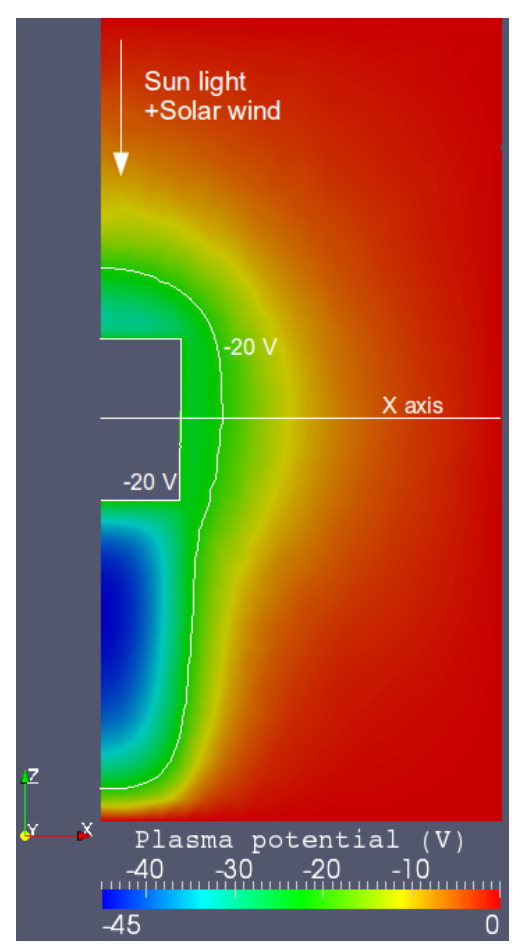

Fig. 5. Cross comparison simulation B (SPIS): Plasma potential in a $X=0$ plane. model has been chosen except for a comparison case that permits to determine the impact of using Boltzmann distribution instead of PIC modelling for electrons. For the ions, the PIC model is used to inject particles following a Maxwellian with an energy of $T_{\mathrm{i}}=82 \mathrm{eV}$ and a drift velocity of $-300 \mathrm{~km} \mathrm{~s}^{-1}$ in the z-direction. This permits to have a more consistent calculation of the plasma state.

The material covering the Solar Probe Plus model is now a conductive layer quite similar to ITO (Indium Tin Oxide). Its SEEY is presented on Fig. 10. Particularly for thermal electrons at $85 \mathrm{eV}$, the backscattering yield of ITO for an isotropic incident flux is 0.18 and the SEE (Secondary Electron Emission) yield of ITO for an isotropic incident flux is 1.63. The secondary electron emission is set with a characteristic energy of $2 \mathrm{eV}$ (Maxwellian distribution). The backscattered electrons are emitted with $2 / 3$ of their initial energy. For photoelectrons and secondary electrons, Debye lengths are expected to be smaller than $5 \mathrm{~cm}$. These conditions justify our choices for the meshing: (1) the smallest grid spacing possible on the sunlit side of the cylinder to compute properly these populations with a PIC model and (2) the intermediate meshing cylinder at $1 \mathrm{~m}$ around the satellite (bigger than $\left.\lambda_{\text {the }}\right)$.

The boundary condition mimics a $1 / r^{2}$ decrease of the potential, which is simulated by a Fourier (or mixed 


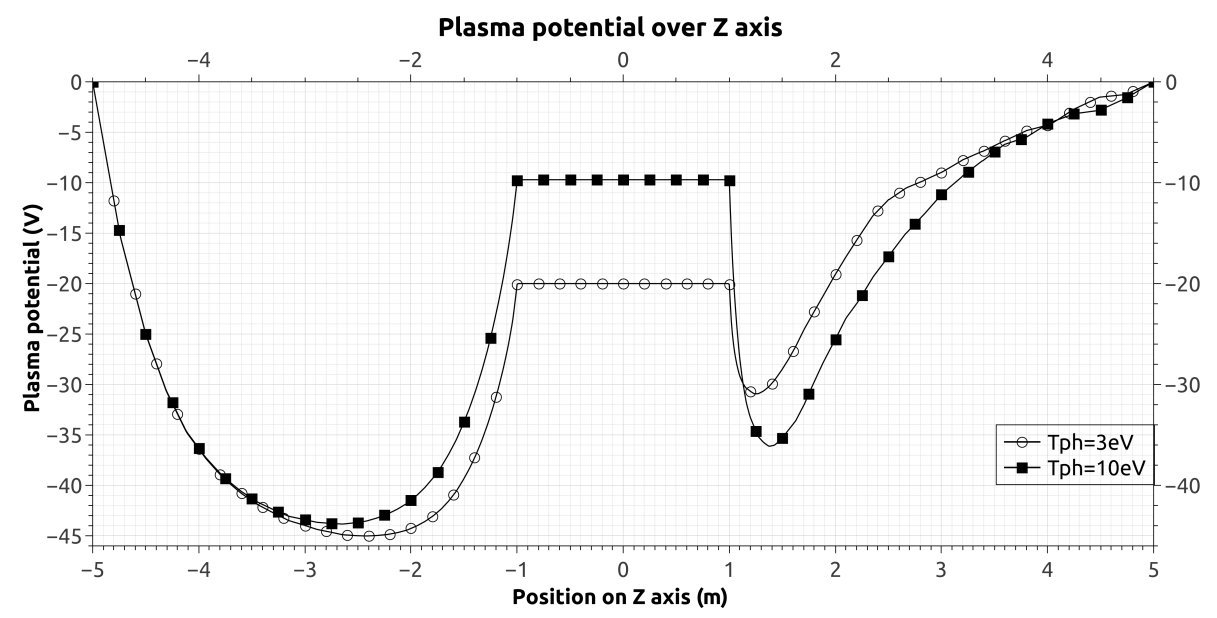

Fig. 6. Cross comparison simulation B (SPIS): plot along the z-axis of the plasma potential. The circled line represents the potential for a photoelectron emission temperature centered on $3 \mathrm{eV}$, the squared line is for a temperature of $10 \mathrm{eV}$.

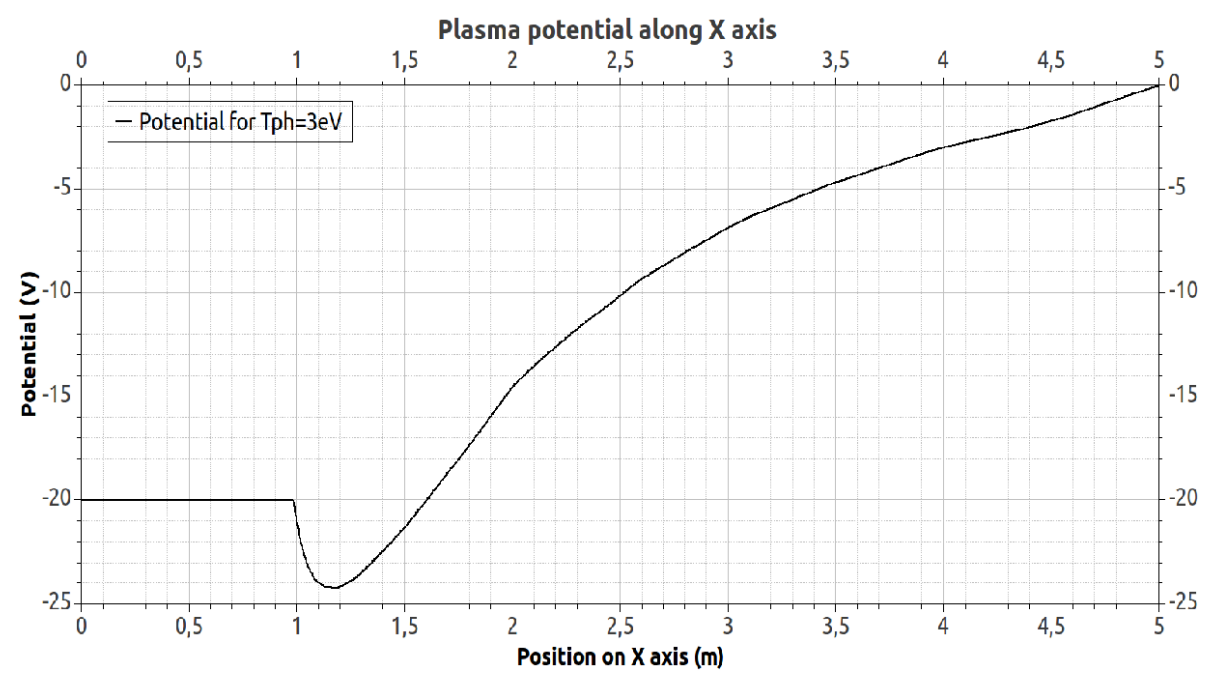

Fig. 7. Cross comparison simulation $\mathrm{B}$ (SPIS): plasma potential along the $\mathrm{x}$-axis, showing the existence of a $-4 \mathrm{~V}$ potential barrier on the side of the spacecraft.

Dirichlet-Neuman) type condition:

$\alpha \phi+\nabla \phi \times \boldsymbol{n}=\beta$

$\alpha=\frac{2 \boldsymbol{r} \times \boldsymbol{n}}{r^{2}}$

with $\beta=0, \boldsymbol{r}$ is the vector field of boundary mesh surface positions with origin at the spacecraft mesh barycentre, and $\boldsymbol{n}$ is the vector field of the outgoing normals to the external boundary mesh.

The parameters common to all simulations ( $\mathrm{S} 1$ to $\mathrm{S} 5, \mathrm{~S} 1$ being the nominal case) are presented in Table 3 . The parameters specific to each case are presented in Table 4. Each non-nominal case (S2 to S5) has only one change with respect to $\mathrm{S} 1$.

\subsection{Results}

The collected, emitted and net final currents of all cases are summarized in Table 5, with all final spacecraft potentials and potential barriers values. In each case the photoemission is constant over time. Figure 11 displays for all cases the plasma potential along the z-axis (for S5: the z-axis is not crossing the center of the wake as a perpendicular spacecraft speed has been added regarding to the Sun-SP+ direction).

\subsubsection{S1: nominal case}

The final spacecraft potential sets up in this S1 case around $-14.5 \mathrm{~V}$ (the plasma potential map is represented on Fig. 12). As previously, two major negative potential barriers for secondary particles are visible: $-10.5 \mathrm{~V}$ in the ram and $-15 \mathrm{~V}$ 


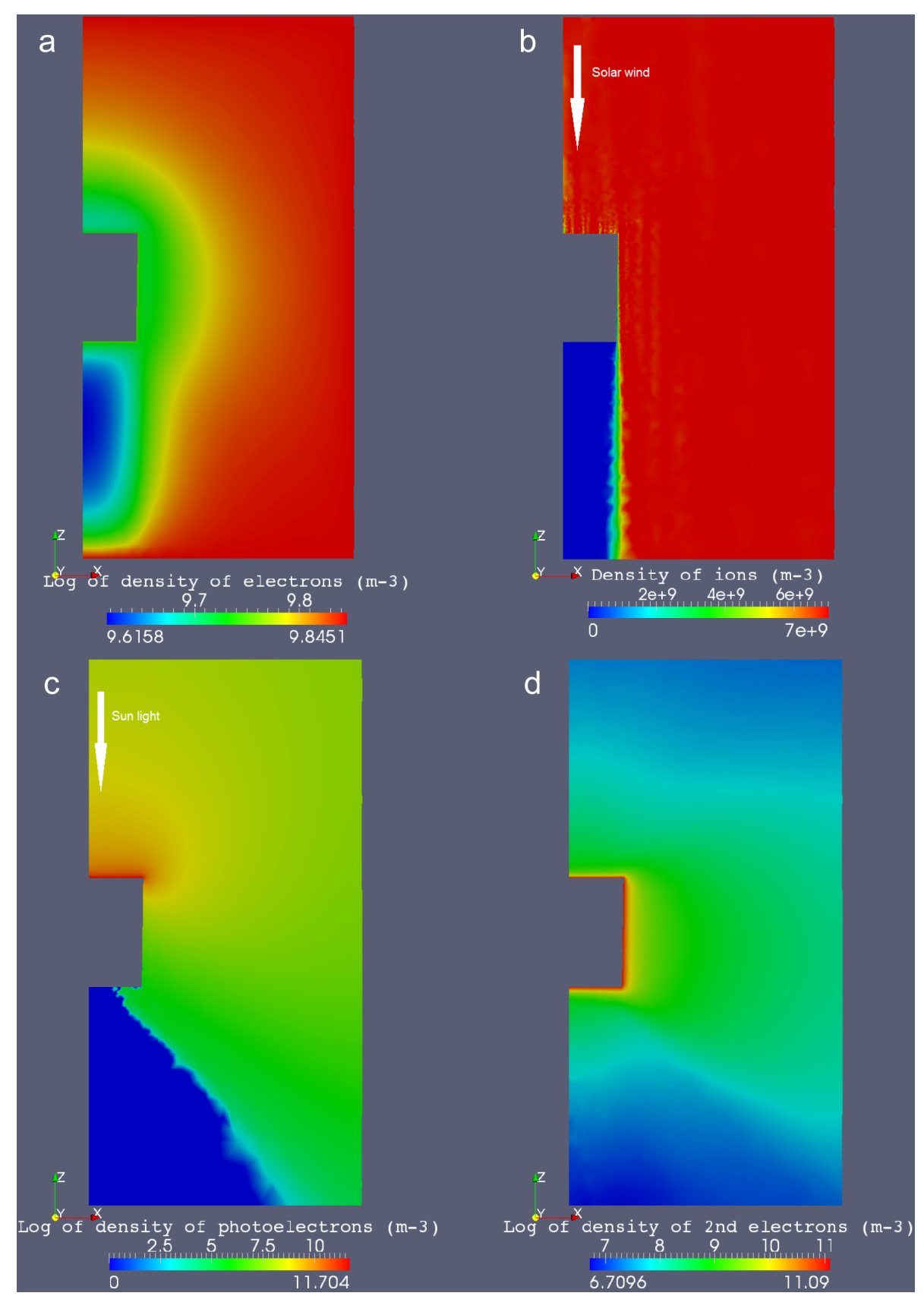

Fig. 8. Cross comparison simulation $B$ (SPIS): population density maps $\left(T_{\mathrm{ph}}=3 \mathrm{eV}\right)$ : $(\mathbf{a}-\mathbf{d})$ from the upper left figure to the lower right figure. (a) Thermal electrons, (b) Ions, (c) Photoelectrons, (d) Secondary electrons.

in the wake. Once the potential is stabilized $76 \%$ of emitted secondary electrons are recollected and this ratio goes up to $92 \%$ for photoelectrons (see Table 5). Globally, the same comments as in the previous section can be made.

The maps in a $X=0$ plane of the particle densities obtained through the S1 simulation are displayed on the Fig. 13. The thermal electron density is almost constant over the simulation box except near the satellite where it goes to $3.16 \times 10^{9} \mathrm{~m}^{-3}$. The ion wake is reduced by the thermal en- ergy of these particles and by ion focussing by the negative potential. Local striations of the ion density plot at the front are due to statistic noise in the ion PIC approach caused by a reduced number of superparticles per cell in this region (it decreases until less than 5). However, that does not impact the results since the space charge is ruled by photoelectron density in the sheath (the space charge used in Poisson solver is computed using charge deposit of ions along their trajectory and not at the end of each time step). Photoelectrons are 


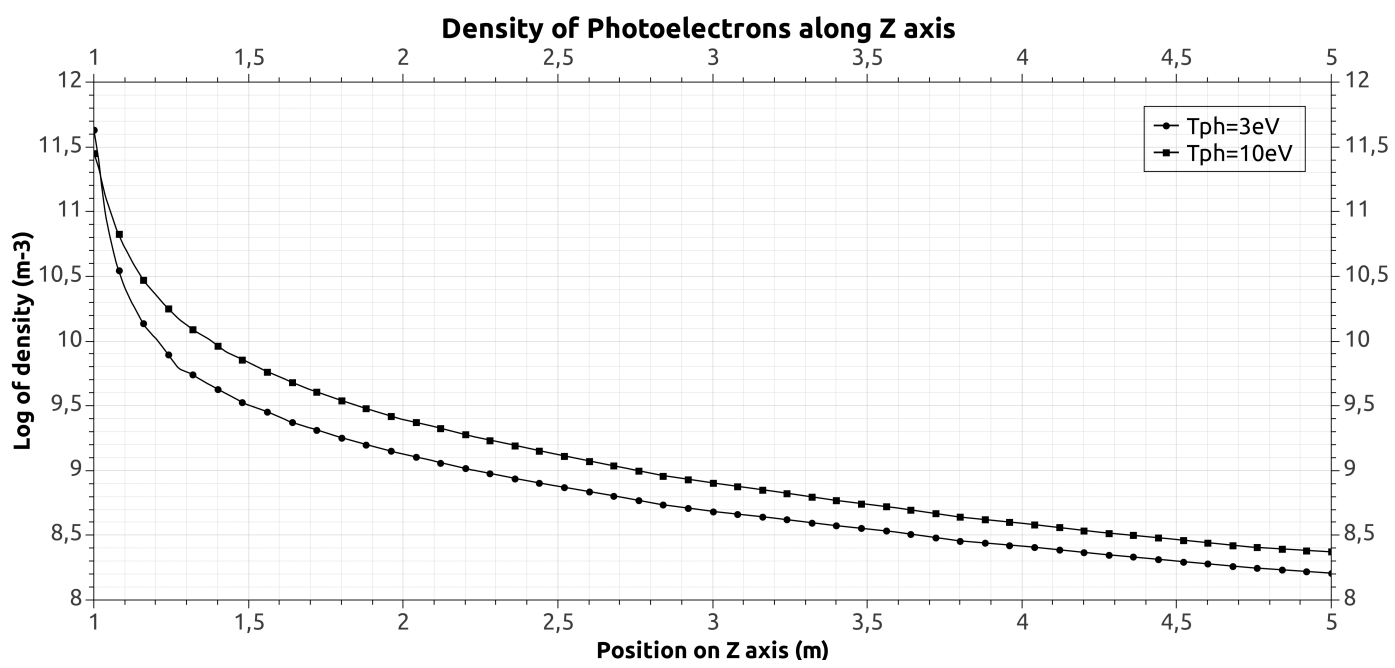

Fig. 9. Cross comparison simulation B (SPIS): plot along the z-axis of the photoelectron density, from the center of the heatshield to the upper boundary of the simulation box. The circled line represents the density for a photoelectron emission temperature of $3 \mathrm{eV}$, the squared line is for a temperature of $10 \mathrm{eV}$.

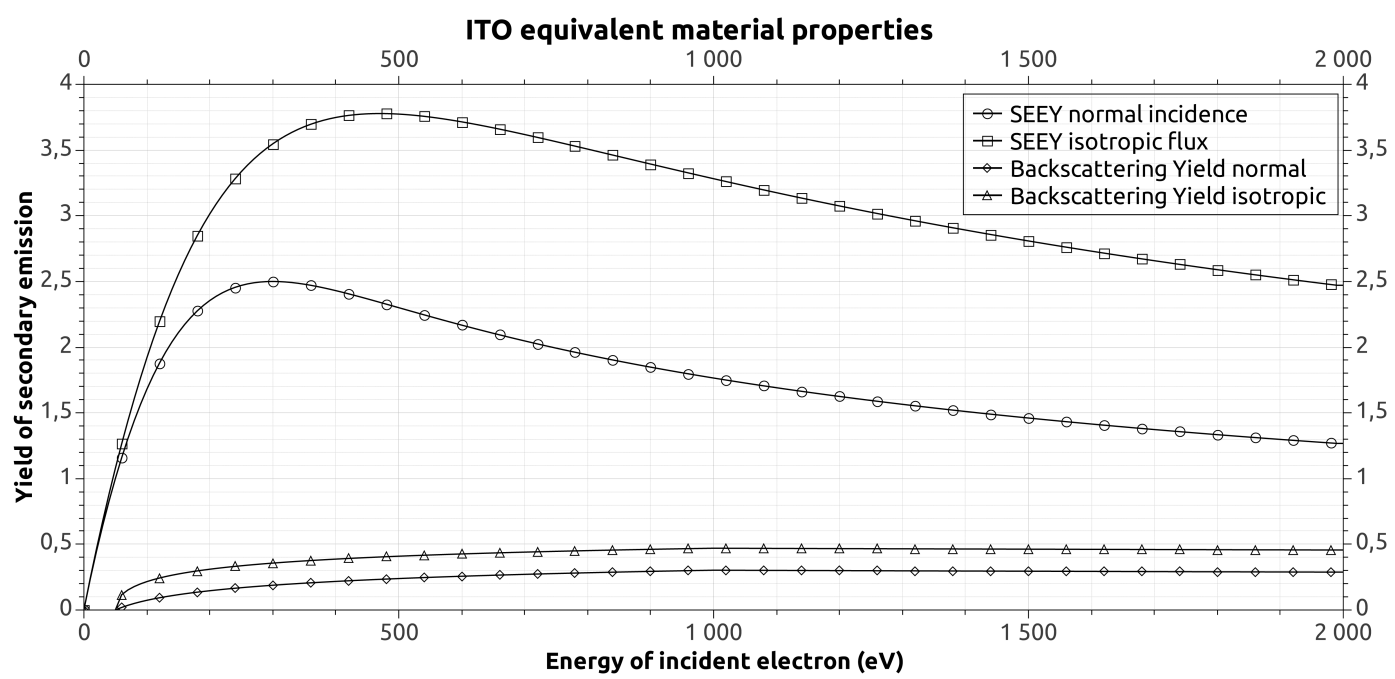

Fig. 10. Secondary electron emission yield (SEEY) and the backscattering yield of ITO material (used in parametric study) vs. incident electron energy (with a normal or isotropic incoming flux).

the denser population: they are emitted from the sunlit face at densities of about $10^{11} \mathrm{~m}^{-3}$ and they are spreading around until the wake zone. The photoelectron wake is also visible on the rear side of the cylinder, and the highly negative potential present there prevents photoelectrons from penetrating this area and from being recollected on this face. Secondary electrons are highly present over all surfaces of the spacecraft, as in the simulation B. The potential barriers still have a great influence by preventing secondary electrons from escaping the front and the back faces of the spacecraft.

Figure 14 shows the ratios of thermal, photo and secondary electrons densities over the plasma density $\left(n_{0}=\right.$ $7 \times 10^{9} \mathrm{~m}^{-3}$ ), the final blue curve being the sum of those con- tributions over $n_{0}$. The photoelectrons and secondary electrons dominate over thermal electrons in the ram, with a higher density of photoelectrons over secondary electrons. Thermal electrons are predominant over secondary electrons $\sim 10 \mathrm{~cm}$ further from the front face and over photoelectrons $\sim 50 \mathrm{~cm}$ further. At the back side of the cylinder, the photoelectrons are not visible because of their extremely low densities with respect to the scale of the plot. The secondary electrons are dominant over thermal ones by $\sim 25 \mathrm{~cm}$.

This $\mathrm{S} 1$ simulation demonstrates that the same phenomena showed in the Sect. 2 occur in a more complex and realistic simulation. One major difference here is the reduced wake dimension due to the considered ion temperature and their 


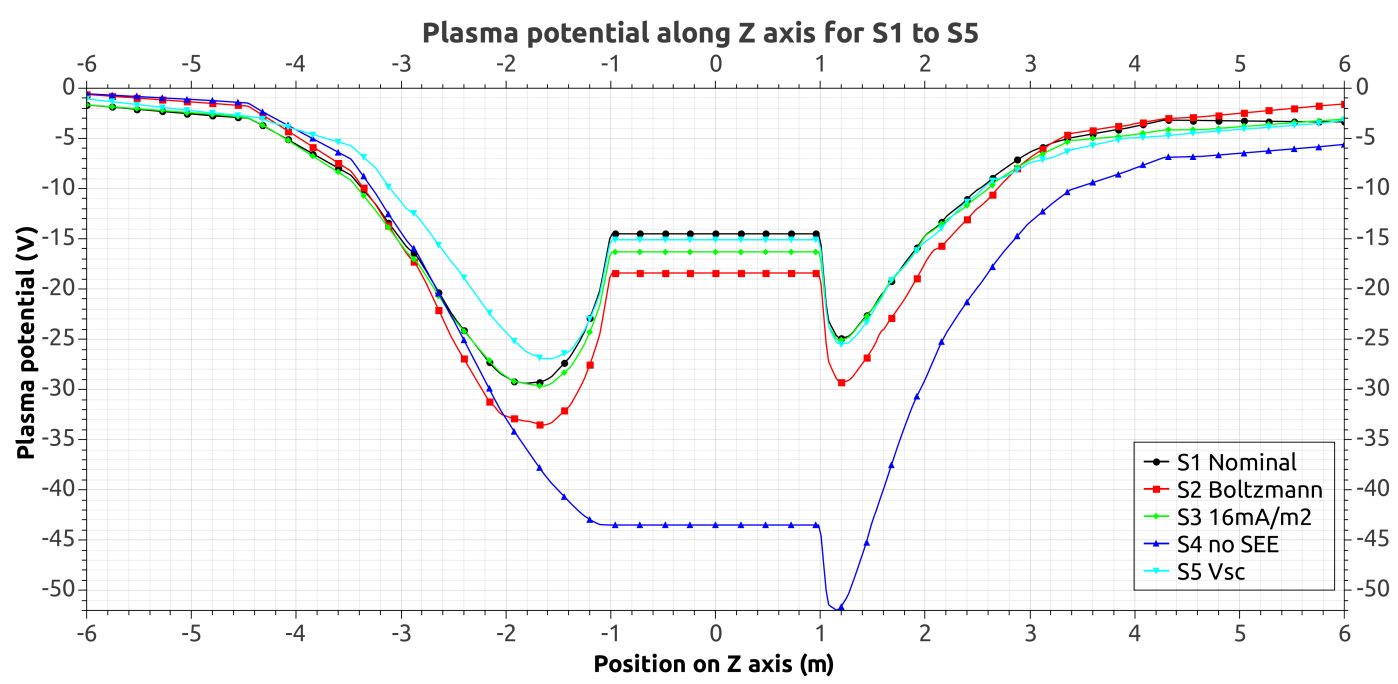

Fig. 11. Plot along the z-axis of the plasma potential for all SPIS simulations (parametric study).

Table 4. Specific inputs for the parametric study.

\begin{tabular}{lllccl}
\hline Simulation & Description & Electron modelling & Ion velocity & $J_{\mathrm{ph}}\left(\mathrm{mA} \mathrm{m}^{-2}\right)$ & 2nd emission \\
\hline S1 & Nominal & PIC & $V_{Z}=-300 \mathrm{~km} \mathrm{~s}^{-1}$ & 29 & Active \\
S2 & Boltzmann & Boltzmann fluid & $V_{Z}=-300 \mathrm{~km} \mathrm{~s}^{-1}$ & 29 & Active \\
S3 & $J_{\mathrm{ph}}=16$ & PIC & $V_{Z}=-300 \mathrm{~km} \mathrm{~s}^{-1}$ & 16 & Active \\
S4 & No SEE & PIC & $V_{Z}=-300 \mathrm{~km} \mathrm{~s}^{-1}$ & 29 & Disabled \\
S5 & Ion drift & PIC & $V_{Z}=-300 \mathrm{~km} \mathrm{~s}^{-1}$ & 29 & Active \\
& & & $V_{X}=-180 \mathrm{~km} \mathrm{~s}^{-1}$ & & \\
\hline
\end{tabular}

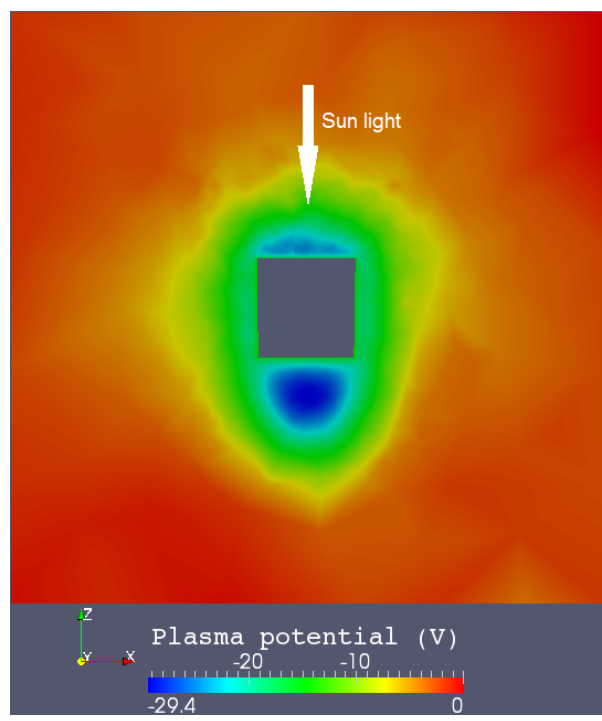

Fig. 12. Simulation S1: map of the plasma potential in a $X=0$ plane. true PIC model: the deflection of their trajectories is possible in this case compared to the previous simulations (A and B). Ions are thus able to spread more efficiently and resettle the wake faster. The reduced wake increases the final probe potential. The ITO coating produces less secondary electrons than the previous material but sufficiently to contribute with photoelectrons to the formation of the potential barriers. Finally, PIC electrons permit to properly account for potential barriers, as it will be demonstrated in the next paragraph.

\subsubsection{S2 simulation: Boltzmann thermal electrons model}

In this $\mathrm{S} 2$ simulation the final spacecraft potential sets up around $-18.4 \mathrm{~V}$, instead of $-14.5 \mathrm{~V}$. Two major negative potential barriers for secondary particles are present (Fig. 11): $-10.6 \mathrm{~V}$ in the ram and $-16.1 \mathrm{~V}$ in the wake. The Boltzmann model for thermal electrons did change the final $\phi_{\mathrm{SC}}$ but not the values of the potential barriers: the whole plasma and satellite potentials have been dug negatively by about $4 \mathrm{~V}$. Indeed the Boltzmann analytical model can not fully describe the physics of potential barriers since it makes the assumption of local thermal equilibrium with $\phi$. The shielding of the low energy thermal electron is however not modelled. The 


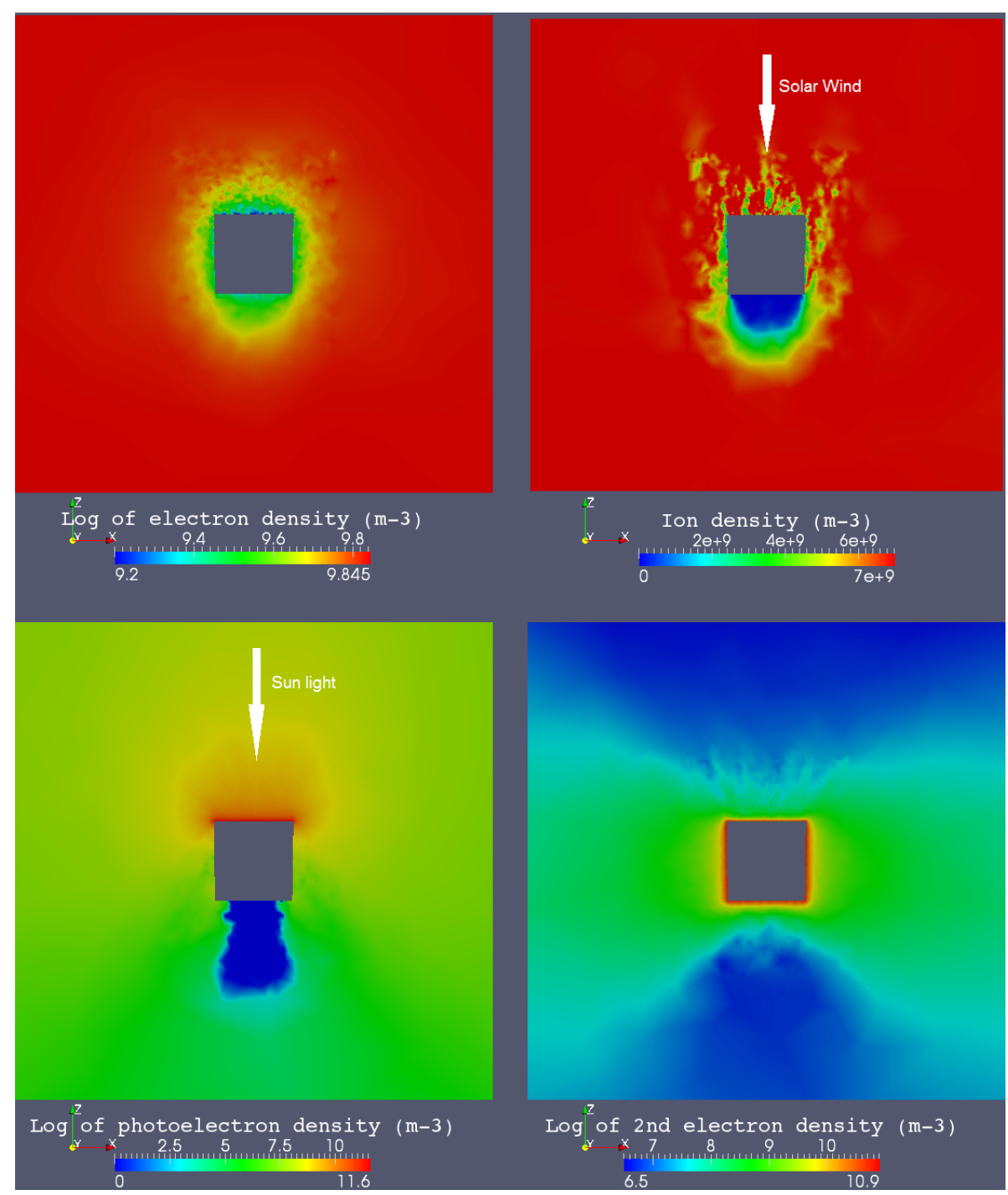

Fig. 13. Simulation S1 population density maps: (a-d) from the upper left figure to the lower right figure. (a) Thermal electrons, (b) Ions, (c) Photoelectrons, (d) Secondary electrons.

surrounding plasma is more negative, which increases the photoelectron recollection and decreases slightly the thermal electron collection (see Table 5). The reduced disturbance on thermal electrons can be seen on Fig. 15.

This type of near-Sun environment requires a PIC thermal electron model for reliable results with precisions under the Volt. The Boltzmann model can be used to get approximate levels of potentials in a shorter computation duration (a gain of time of $\sim 50 \%$ with this simulation), in order to prepare full PIC simulations.

\subsubsection{S3 simulation: effect of reduced photoemission}

As discussed previously, Ergun et al. (2010) simulations are based on a photoelectron emission yield of $29 \mathrm{~mA} \mathrm{~m}^{-2}$. An equivalent material has been previously chosen as conductive layer covering the satellite structure and the solar flux intensity was adapted to reach this photoelectron yield. However, with the solar flux intensity at $0.044 \mathrm{AU}$ and a ITO surface, SPIS computes a $J_{\mathrm{ph}}$ of $\sim 16 \mathrm{~mA} \mathrm{~m}^{-2}$, almost half of the rate supposed in the previous A and B cases. The S3 simulation checks the potential barriers settlement in case of this reduced photoemission.

The final $\phi_{\mathrm{SC}}$ is set at $-16.3 \mathrm{~V}$, which is 2 Volts lower than for S1. However, the plasma potential around the probe is unchanged regarding to the S1 case (see Fig. 11), the ram and wake barriers for particles are thus slightly inferior than in S1 but the recollection rates are similar: $88 \%$ for photoelectrons and $74 \%$ for secondary electrons. The immediate effect of $J_{\mathrm{ph}}=16 \mathrm{~mA} \mathrm{~m}^{-2}$ instead of $29 \mathrm{~mA} \mathrm{~m}^{-2}$ is that emitted and recollected currents due to photoelectrons are divided by almost 2: respectively -44 and $-50 \mathrm{~mA}$ (instead of 


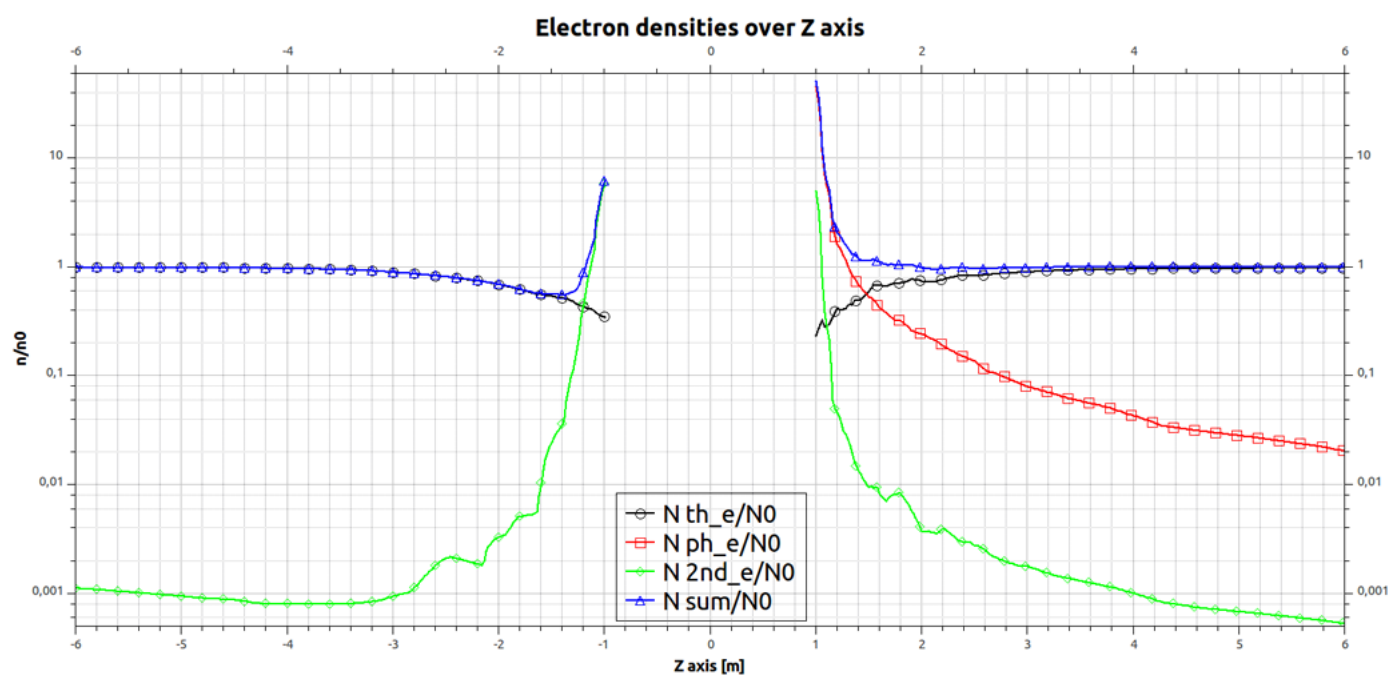

Fig. 14. Simulation S1: plot along the z-axis of electrons over $n_{0}$, the plasma density: $7 \times 10^{9} \mathrm{~m}^{-3}$.

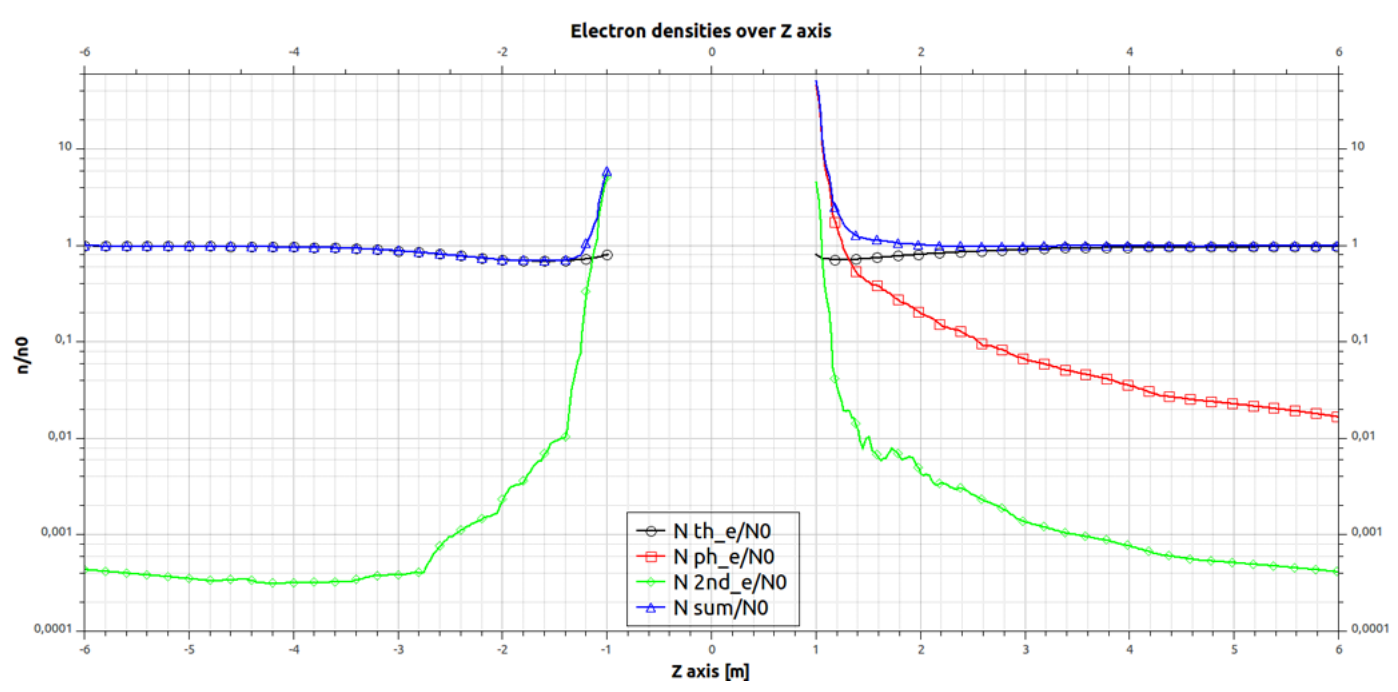

Fig. 15. Simulation $\mathrm{S} 2$ : plot along the $\mathrm{z}$-axis of electrons over $n_{0}$, the plasma density: $7 \times 10^{9} \mathrm{~m}^{-3}$.

-84 and $-91 \mathrm{~mA}$ for $\mathrm{S} 1)$. Other currents are almost not affected (Table 5). This 2 Volts lower final $\phi_{\mathrm{SC}}(12 \%$ of difference regarding the $\mathrm{S} 1 \phi_{\mathrm{SC}}$ of $-14.5 \mathrm{~V}$ ) is a consequence of a $50 \%$ reduced photoemission. The conclusion, based also on the cross-comparison simulation results, is that the photoemission yield and the characteristic emission temperature of photoelectrons are highly important in this specific environment.

\subsubsection{S4 simulation: no secondary electronic emission}

As the emission of secondary electrons under thermal electron impact is highly dependent on the type of materials covering the satellite, a S4 simulation was generated with no secondary emission to observe the behaviour of the spacecraft and its close environment in this extreme situation. In previous simulations each thermal electron impact liberates in average $\sim 1.5$ secondary electron.

This time $\phi_{\mathrm{SC}}$ sets up at $-43.5 \mathrm{~V}$ (because the spacecraft is not emitting electrons anymore), and the surrounding plasma is also highly affected by this changed parameter: ram and wake regions reach values of -52 and $-43.5 \mathrm{~V}$ (Fig. 11), respectively. The thermal electron collection is thus decreased $(-19 \mathrm{~mA}$ compared to $\sim-26 \mathrm{~mA}$ before), and the reduced ram barrier for photoelectrons $(-8.5 \mathrm{~V})$ allows them to escape more efficiently (recollection rate of $79 \%$ instead of about $90 \%$ in previous simulations). The population maps for S1 on Fig. 13 showed that those particles should be present in this region with densities between $10^{9}$ and $10^{6} \mathrm{~m}^{-3}$, digging the plasma potential and generating a wake barrier for secondaries. Here there is no SEE to produce a potential barrier anymore (see Fig. 11). 


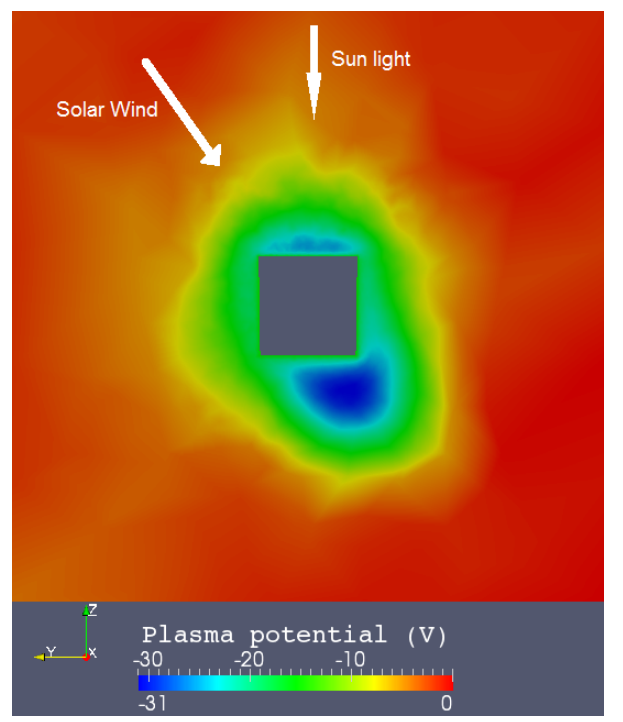

Fig. 16. Simulation S5: map of the final plasma potential in a $X=0$ plane.

This extreme case showed that the secondary emission yield has a great influence on the spacecraft and surrounding plasma potential. As Solar Probe Plus will not be covered with only one single material with a specific emission yield, more precise information on the different layers properties are needed to investigate properly the effects of the near-Sun environment on the electric fields and secondary particles.

\subsubsection{S5 simulation: spacecraft drift velocity}

In this S5 case, a spacecraft speed component perpendicular to the solar wind speed is added (simulating an ion velocity of $-180 \mathrm{~km} \mathrm{~s}^{-1}$ in the y-direction.) to verify the effects of an aside shifted wake behind the spacecraft. This corresponds to one part of the predicted orbit of Solar Probe Plus at this distance to the Sun.

The spacecraft potential decreases until $-15.1 \mathrm{~V}$, instead of $-14.5 \mathrm{~V}$ for the $\mathrm{S} 1$ case. This result is practically the same for $\mathrm{S} 1$ (showing that the final spacecraft potential is not really affected by a perpendicular ion velocity of $180 \mathrm{~km} \mathrm{~s}^{-1}$ ). The global values of plasma potential and barriers are practically unchanged, it is just the position of the wake that is shifted aside, as represented on the plasma potential map Fig. 16. The values for S5 plasma potentials in Table 5 are truly measured along the wake axis and are practically equal to the values for S1. In the center of the ion wake the potential is $4 \mathrm{~V}$ lower than on the $\mathrm{z}$-axis in this region. Neither the front area potential nor the final currents are affected by the spacecraft drift velocity. But the shifted wake is setting up an asymmetry of the plasma potential against the zaxis, as it appears clearly on Fig. 18, representing the plasma population map densities. The plasma populations densities are matching the plasma potential map and the shifted ion wake, except for thermal electrons. Looking at the secondary electrons, the shifted wake and the higher potential barrier set on the $-\mathrm{Y}$ side of the spacecraft enhance the acceleration process: the emitted particles that were not recollected could spread along the structure in the $+\mathrm{Z}$ region leading to densities of $\sim 10^{9} \mathrm{~m}^{-3}$ near the side of the spacecraft. However, on the $-\mathrm{Y}$ side, those secondary electrons encounter a local potential of $-21 \mathrm{~V}$ that rejects them (density in this region reach $\sim 10^{7}-10^{8} \mathrm{~m}^{-3}$ ). In Fig. 11 , the $\mathrm{z}$-axis is not crossing the center of the shifted wake so the real potential along the wake axis is deeper. Further analysis of the potential map shows that the potential barrier by the $-\mathrm{Y}$ side of the cylinder (the one non impacted by ion side) is deeper: $\delta \phi_{2 \text { nd }}(-\mathrm{Y})=-5 \mathrm{~V}$ while $\delta \phi_{2 \text { nd }}(+\mathrm{Y})=-3 \mathrm{~V}$ due to the arrival of positively charged ions. This is showed on Fig. 17, which displays the plasma potential over the y-axis (from +6 to $-6 \mathrm{~m}$ with respect to the potential map Fig. 16): the ions have a $\mathrm{Y}$ velocity component from the left side of the plot to the right. Thus, the recollection of secondary electrons on the $-\mathrm{Y}$ side of the satellite is slightly enhanced. Comparing to $\mathrm{S} 1$ this time the negative wake is reducing the possibility of secondaries to escape through the $-\mathrm{Y}$ side. A wider and bigger flux of those particles can be seen on the $+Y$ side of $\mathrm{SP}+$ on the Fig. 18. This effect appears less clearly for photoelectrons but it also exists.

The shifted wake did not change significantly the spacecraft potential. However, the global plasma behaviour lost its symmetry around the z-axis and the near plasma potential is different whether we look on the exposed to ions side of the spacecraft or not. A shifted wake may potentially complicate particle measurements, as electron instruments are indeed placed on the side of Solar Probe Plus.

\section{Conclusion and perspectives}

The near-Sun environment effect picture is confirmed in this paper. Indeed, main phenomenon previously predicted and simulated with simple models are here achieved through different PIC numerical codes: the spacecraft structure tends to settle at a negative potential (of typical -10 to $-20 \mathrm{~V}$ ), due to the surrounding presence of electrostatic barriers originating from secondary electrons and photoelectrons which bring back the secondary particles to the spacecraft. A more realistic modelling gives better accuracy on the spacecraft charging levels obtained. The parametric study using SPIS achieved the same phenomena and furthermore emphasises the importance of key parameters, that heavily affect, respectively, the final Solar Probe Plus potential and the surrounding plasma potential near the probe. The photoelectron emission temperature and yield are important for the final spacecraft potential. The three controlling parameters that require more investigations are (1) the photoelectron temperature, (2) the secondary electron emission yield (also depending on the coating materials) and (3) the orientation of 


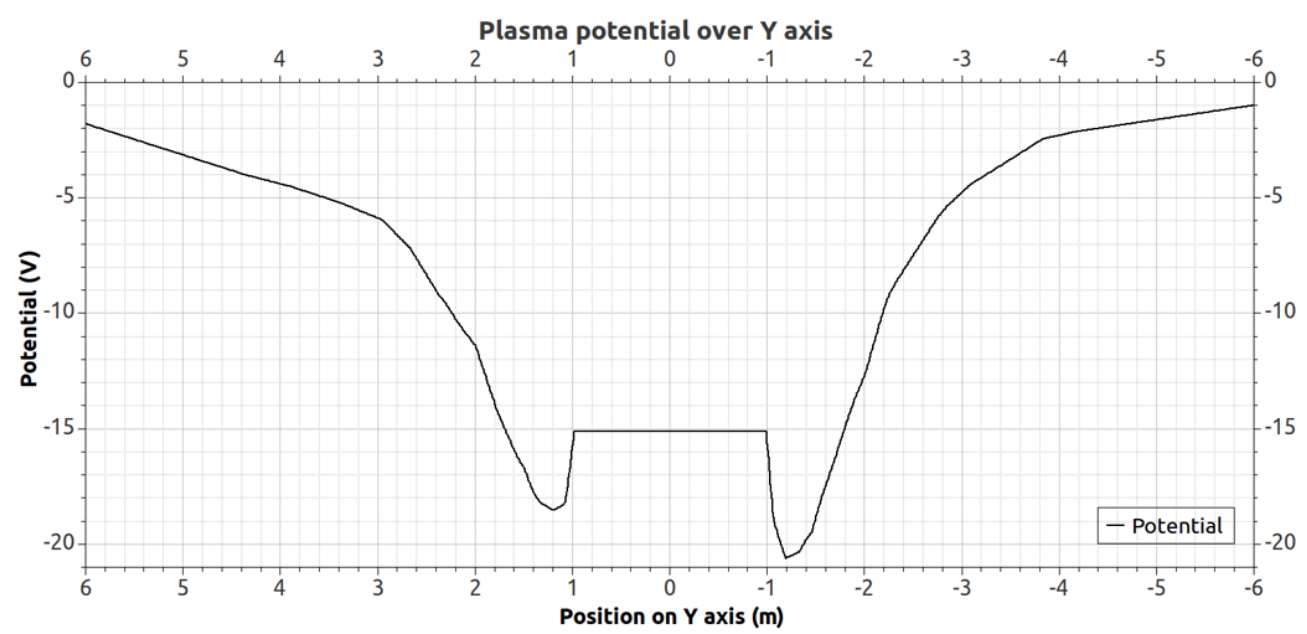

Fig. 17. Simulation S5: plot along the y-axis of the plasma potential. From +6 to $-6 \mathrm{~m}$ with respect to the potential map Fig. 16 : the ions have a $\mathrm{Y}$ velocity component from the left side of the plot to the right.

Table 5. Final currents and potentials for all S cases. Potential barriers are calculated for secondary particles emitted from the spacecraft (photoelectrons and secondary electrons).

\begin{tabular}{llccccc}
\hline Currents (mA) & Studied Value & S1 Nominal & S2 Boltzmann & S3 $J_{\mathrm{ph}} / 2$ & S4 No SEE & S5 $V_{\mathrm{sc}}$ \\
\hline Ions & Collected & 2.1 & 2 & 1.3 & 1.7 & 1.7 \\
\hline Electrons & Collected & -26.7 & -25.5 & -26.2 & -19.2 & -26.5 \\
\hline Photoelectrons & Collected & -84.1 & -85.2 & -44.3 & -72.4 & -83.8 \\
& Emitted & -91.1 & -91.1 & -50 & -91.1 & -91.1 \\
& Net (\% recollection) & $7(92 \%)$ & $5.9(93 \%)$ & $5.7(88 \%)$ & $18.7(79 \%)$ & $7.3(92 \%)$ \\
\hline \multirow{2}{*}{ nd electrons } & Collected & -52.3 & -49.1 & -49.4 & 0 & -52.8 \\
& Emitted & -68.6 & -65.6 & -67.2 & 0 & -68 \\
& Net (\% recollection) & $16.3(76 \%)$ & $16.5(75 \%)$ & $17.8(74 \%)$ & 0 & $15.2(78 \%)$ \\
\hline \multirow{2}{*}{ All populations } & Collected & -161 & -157.8 & -118.6 & -89.9 & -161.4 \\
& Emitted & -159.7 & -156.7 & -117.4 & -91.1 & -159.1 \\
& Net & -1.3 & -1.1 & -1.2 & 1.2 & -2.3 \\
\hline \multirow{2}{*}{$(\mathrm{V})$} & Spacecraft & -14.5 & -18.4 & -16.3 & -43.5 & -15.1 \\
& Ram & -25 & -29 & -25 & -52 & -25.5 \\
& Wake & -29.5 & -34.5 & -29.5 & -43.5 & -31 \\
\hline & Ram barrier & -10.5 & -10.6 & -8.7 & -8.5 & -10.4 \\
& Wake barrier & -15 & -16.1 & -13.2 & 0 & -15.9 \\
\hline
\end{tabular}

the wake (potentially modifying the plasma measurements depending on the position of the instruments regarding to the ion flux). This is necessary to at least take into account full PIC modelling and good models of photoelectron and secondary SEEE. The photoelectron temperature study will need a more realistic model of photoemission to be implemented in the SPIS numerical core. The secondary particles recollection is problematic for the plasma instruments, especially the secondary electrons recollection which can occur all around the spacecraft, as it was demonstrated in all previous SPIS simulations. Those final negative spacecraft potentials will definitely affect low energy plasma measurements, and further investigations are needed to quantify precisely the fraction of the ambient Solar wind electrons that will be missed by the electron instruments and the impacts of this charging on the onboard plasma moment computation.

To reach very good previsions, SPIS new developments will focus on: ambient population distributions, material data, detector modelling and boundary conditions (presentation of new SPIS capabilities are displayed in Matéo-Vélez et al., 2012b). In this context, further studies with more complex models of Solar Orbiter are now under way. Indeed, it was showed in Isensee (1977) that regions as far as $0.2 \mathrm{AU}$ from the Sun are not spared by the ram and wake potential 


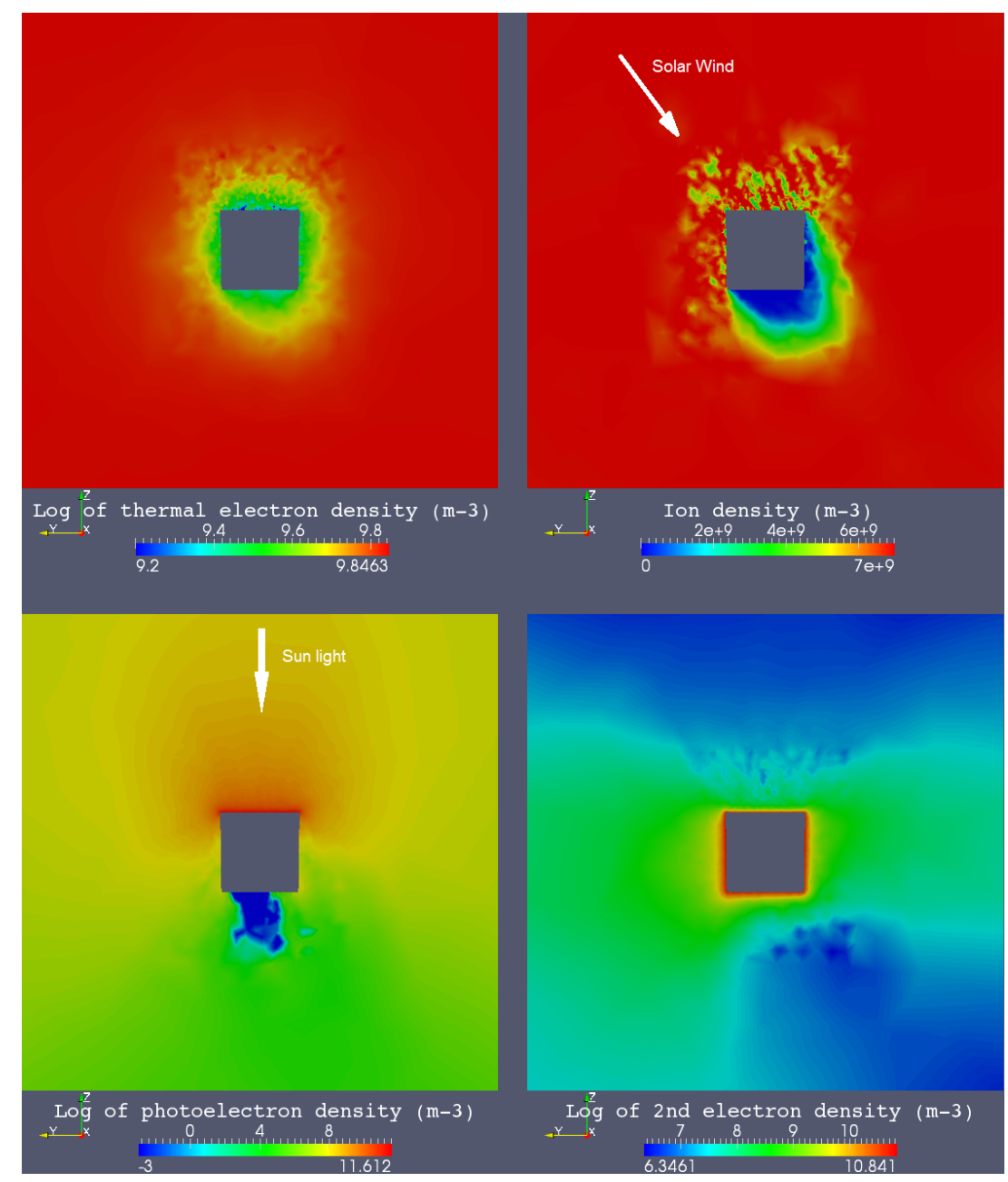

Fig. 18. Simulation S5 population density maps: (a-d) from the upper left figure to the lower right figure. (a) Thermal electrons, (b) Ions, (c) Photoelectrons, (d) Secondary electrons.

barriers, even if their depths are less important. Preliminary results (Guillemant et al., 2012) show that ram/wake potential barriers can also appear between 0.25 and $0.3 \mathrm{AU}$ from the Sun, leading to investigate the Solar Orbiter perihelion (at $0.28 \mathrm{AU}$ ). An other important development would consist in modelling a hot electron population within the plasma (the so called Solar wind non thermal populations "Halo" and "Strahl"), and check the potentially increasing charging effects on the spacecraft. Indeed using data from Helios (M. Maksimovic, personal communication, 2011) and associated modelling (Stverak et al., 2009), it is possible to obtain the different electron population contributions in the distribution function by extrapolating results at Helios orbit to other distances from the Sun. We will use this analysis to set up SPIS with a more detailed distribution function for the ambient electron at the Solar Orbiter perihelion. The onboard instruments, especially the SWA-EAS (Solar Wind AnalyserElectron Analyser System), will be modelled and simulated. The associated measurements will be also simulated to deter- mine the impacts of the possible potential barriers and charging effects on the particle moments calculation.

Acknowledgements. The authors wish to thank Alain Hilgers (ESA, ESTEC) for being one of the initiator of the SPIS concept and project at ESA, for giving useful advice at the beginning of this work, and finally for reviewing the manuscript. We also acknowledge the support of the ISSI (International Space Science Institute) team "Spacecraft interaction with space environment", led by Richard Marchand (University of Alberta, Canada) and its members for their interest and precious advices for our work. We also thank the French Centre National d'Etudes Spatiales (CNES) and the Région Midi-Pyrénées for providing the scholarship for this Ph.D.

Topical Editor R. Nakamura thanks C. Cully and M. Maksimovic for their help in evaluating this paper. 


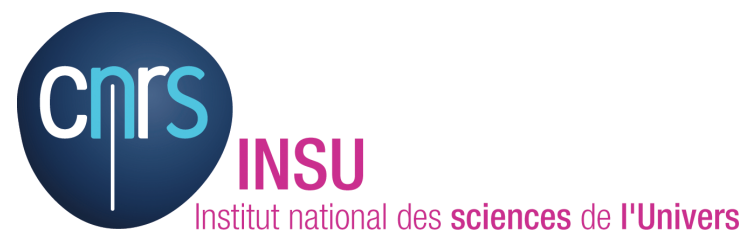

The publication of this article is financed by CNRS-INSU.

\section{References}

Ergun, R. E., Malaspina, D. M., Bale, S. D., McFadden, J. P., Larson, D. E., Mozer, F. S., Meyer-Vernet, N., Maksimovic, M., Kellogg, P. J., and Wygant, J. R.: Spacecraft charging and ion wake formation in the near-Sun environment, Phys. Plasmas, 17, 1134-1150, 2010.

Guillemant, S., Génot, V., Matéo-Vélez, J.-C., and Louarn, P.: Scientific spacecraft cleanliness: influence of heliocentric distance, in: Proceedings of 12th Spacecraft Charging Technology Conference, Kitakyushu, Japan, 14-18 May 2012.

Hilgers, A., Thiébault, B., Estublier, D., Gengembre, E., Gonzalez, J., Tajmar, M., Roussel, J.-F., and Forest, J.: A simple model of SMART-1 electrostatic potential variation, IEEE Trans. Plasma Sci., 34, 2159-2165, doi:10.1109/TPS.2006.883405, 2006.

Hilgers, A., Clucas, S., Thiébault, B., Roussel, J.-F., MatéoVélez, J.-C., Forest, J., and Rodgers, D.: Modelling of Plasma Probe Interactions With a PIC Code Using an Unstructured Mesh, IEEE Trans. Plasma Sci., 36, 2319-2323, doi:10.1109/TPS.2008.2003360, 2008.

Isensee, U.: Plasma disturbances caused by the Helios spacecraft in the Solar Wind, J. Geophys., 42, 581-589, 1977.

Lai, S. T.: Theory and Observation of Triple-Root Jump in Spacecraft Charging, J. Geophys. Res., 96, 19269, doi:10.1029/91JA01653, 1991.

Lipatov, A. S., Sittler, E. C., Hartle, R. E., and Cooper, J. F.: The Interaction of the Solar Wind with Solar Probe Plus - 3D Hybrid Simulation, Report 2: The study for the distance $9.5 R_{\mathrm{S}}$, NASA/TM 2010-215863, 2010.

Matéo-Vélez, J.-C., Roussel, J.-F., Sarrail, D., Boulay, F., Inguimbert, V., and Payan, D.: Ground Plasma Tank Modeling and Comparison to Measurements, IEEE Trans. Plasma Sci., 36, 23692377, doi:10.1109/TPS.2008.2002822, 2008.

Matéo-Vélez, J.-C., Roussel, J.-F., Inguimbert, V., Cho, M., Saito, K., and Payan, D.: SPIS and MUSCAT software comparison on LEO-like environment, IEEE Trans. Plasma Sci., 40, 177-182, 2012a.
Matéo-Vélez, J.-C., Sarrailh, P., Thiebault, B., Forest, J., Hilgers, A., Roussel, J.-F., Dufour, G., Rivière, B., Génot, V., Guillemant, S., Eriksson, A., Cully, C., and Rodgers, D.: SPIS Science: modelling spacecraft cleanliness for low-energy plasma measurement, in: Proceedings of 12th Spacecraft Charging Technology Conference, Kitakyushu, Japan, 14-18 May 2012b.

Pedersen, A.: Solar wind and magnetosphere plasma diagnostics by spacecraft electrostatic potential measurements, Ann. Geophys., 13, 118-129, doi:10.1007/s00585-995-0118-8, 1995.

Roussel, J.-F., Rogier, F., Dufour, G., Mateo-Velez, J.-C., Forest, J., Hilgers, A., Rodgers, D., Girard, L., and Payan, D. SPIS Open Source Code: Methods, Capabilities, Achievements and Prospects, IEEE Trans. Plasma Sci., 36, 2360-2368, doi:10.1109/TPS.2008.2002327, 2008a.

Roussel, J.-F., Tondu, T., Matéo-Vélez, J.-C., Chesta, E., D'Escrivan, S., and Perraud, L.: Modeling of FEEP Electric Propulsion Plume Effects on Microscope Spacecraft, IEEE Trans. Plasma Sci., 36, 2378-2386, doi:10.1109/TPS.2008.2002541, 2008b.

Roussel, J.-F., Dufour, G., Matéo-Vélez, J.-C., Thiébault, B., Andersson, B., Rodgers, D., Hilgers, A., and Payan, D.: SPIS multitime scale and multi physics capabilities: development and application to GEO charging and flashover modeling, IEEE Trans. Plasma Sci., 40, doi:10.1109/TPS.2011.2177672, in press, 2012.

Sarrailh, P., Matéo-Vélez, J.-C., Roussel, J.-F., Dirassen, B., Forest, J., Thiébault, B., Rodgers, D., and Hilgers, A.: Comparison of numerical and experimental investigations on the ESD onset in the Inverted Potential Gradient situation in GEO, IEEE Trans. Plasma Sci., 40, 368-379, 2010.

Štverák, Š., Maksimovic, M., Trávníček, P. M., Marsch, E., Fazakerley, A. N., and Scime, E. E.: Radial evolution of nonthermal electron populations in the low-latitude solar wind: Helios, Cluster, and Ulysses Observations, J. Geophys. Res., 114, A05104, doi:10.1029/2008JA013883, 2009.

Thiebault, B., Hilgers, A., Sasot, E., Laakso, H., Escoubet, C. P., Génot, V., and Forest, J.: Potential barrier in the electrostatic sheath around a magnetospheric spacecraft, J. Geophys. Res., 109, A12207, doi:10.1029/2004JA010398, 2004.

Whipple Jr., E. C.: Theory of spherically symmetric photoelectron sheath: a thick sheath approximation and comparison with the ATS 6 observation of a potential barrier, J. Geophys. Res., 81, 601-607, 1976.

Zhao, H., Schmidt, R., Escoubet, C. P., Torkar, K., and Riedler, W.: Self-consistentdetermination of the electrostatic potential barrier due to the photoelectron sheath near a spacecraft, J. Geophys. Res., 101, 15653-15659, 1996. 